\title{
POSITIVE PLÜCKER TREE CERTIFICATES FOR NON-REALIZABILITY
}

\author{
JULIAN PFEIFLE
}

\begin{abstract}
We introduce a new method for finding a non-realizability certificate of a simplicial sphere $\Sigma$ : we exhibit a monomial combination of classical 3-term Plücker relations that yields a sum of products of determinants that are known to be positive in any realization of $\Sigma$; but their sum should vanish, contradiction. Using this technique, we prove for the first time the non-realizability of a balanced 2-neighborly 3-sphere constructed by Zheng, a family of highly neighborly centrally symmetric spheres constructed by by Novik and Zheng, and several combinatorial prismatoids introduced by Criado and Santos. The method in fact works for orientable pseudo-manifolds, not just for spheres.
\end{abstract}

\section{Introduction}

We start with an overview in which we assume familiarity with all the terms used, and define them all later. For now, recall that a simplicial $(d-1)$-sphere $\Sigma$, given as a list of facets, is polytopal if it occurs as the boundary complex of some simplicial convex $d$-polytope. We suspect that deciding whether such a polytope actually exists is very hard, and in some cases to be discussed later we know this to be true.

We present a new technique for finding final polynomials [BS89, Theorem 4.16] for deciding this question. These certificates consist of polynomial combinations of Plücker relations on $\Sigma$ such that the result is manifestly positive in any realization of $\Sigma$. The existence of such a certificate proves non-realizability, because the Plücker relations vanish on any realization of $\Sigma$, and so cannot combine to a positive number.

1.1. A minimal working example. Sometimes just one relation suffices to prove non-realizability.

Example 1.1. The following list of facets defines a non-realizable 3 -sphere $\Sigma$ with $f$-vector $(8,27,38,19)$ :

$$
\begin{aligned}
& +[0123]-[0124]+[0135]-[0146]+[0157]-[0167]-[0234]+[0345]-[0456]+[0567] \\
& +[1237]-[1246]-[1267]+[1357]-[2347]+[2456]-[2457]-[2567]+[3457]
\end{aligned}
$$

The signs before the facets turn this list of simplices into a simplicial cycle representing the top homology class of this 3-sphere. Now suppose we have a realization of $\Sigma$, and consider the 3-term Plücker relation

$$
0=\Gamma(045 \mid 1267)=[04512][04567]-[04516][04527]+[04517][04526] .
$$

By permuting the entries inside the determinants and changing the sign accordingly, we obtain

$$
(-1)[01425] \cdot(-1)[05674]-[04651] \cdot(-1)[24750]+[01574] \cdot[24560]=0,
$$

where now all determinants are positive.

For example, [01425] $>0$ because [01425] $=-[01245]$, and [01245] is the "signed slack" of $x_{5}$ with respect to the facet [0124] in the supposed convex realization of $\Sigma$; but the orientation of 0124 in $\Gamma$ is negative by the above list. The other determinants can be similarly checked to be positive.

We have obtained a sum of three positive numbers that sums to zero, contradicting the realizability of $\Sigma$. Using the notation of Definition 3.2 and Convention 3.3 introduced later on, the certificate (1.1) reads

$$
\Gamma(045 \mid 1267)=[0142 \mid 5][0567 \mid 4]+[0465 \mid 1][2475 \mid 0]+[0157 \mid 4][2456 \mid 0]=0 .
$$

Date: December 21, 2020.

The author was supported by the grant PID2019-106188GB-I00 from the Spanish Ministry of Education (MEC). 
1.2. Jockusch's centrally symmetric 3 -sphere on 12 vertices. Novik and Zheng [NZ19, NZ20] recently constructed several families of centrally symmetric simplicial 3-spheres. [NZ19, Problem 5.1] asks for properties of these spheres that pertain to realizability. As a first application of our method, we prove the nonpolytopality of the simplicial 3 -sphere $\Delta_{6}^{3}$ with 12 vertices and 48 facets which underlies their construction. This sphere was first constructed by Jockusch [Joc95] as part of an infinite family $\left\{\Delta_{n}^{3}: n \geq 4\right\}$.

Proposition 1.2. Jockusch's 3-sphere $\Delta_{6}^{3}$ is not polytopal.

Proof. An orientation of this 3 -sphere consists of the following oriented facets, where $\bar{x}=-x$ :

$$
\begin{aligned}
& +[1256]+[\overline{1} \overline{2} \overline{5} \overline{6}]+[\overline{1} \overline{2} 56]+[12 \overline{5} \overline{6}]+[2356]+[\overline{2} \overline{3} \overline{5} \overline{6}]+[\overline{2} \overline{3} 56]+[23 \overline{5} \overline{6}]+[3456]+[\overline{3} \overline{4} \overline{5} \overline{6}]+[\overline{3} \overline{4} 56] \\
& +[34 \overline{5} \overline{6}]-[1 \overline{4} 56]-[\overline{1} 4 \overline{5} \overline{6}]+[1 \overline{4} \overline{5} 6]+[\overline{1} 45 \overline{6}]-[1 \overline{4} \overline{5} \bar{b}]-[\overline{1} 456]+[1235]+[\overline{1} \overline{2} \overline{3} \overline{5}]+[\overline{1} \overline{2} 35]+[12 \overline{3} \overline{5}] \\
& -[1 \overline{2} 35]-[\overline{1} 2 \overline{3} \overline{5}]-[1246]-[\overline{1} \overline{2} \overline{4} \overline{6}]-[\overline{1} \overline{2} 46]-[12 \overline{4} \overline{6}]-[2346]-[\overline{2} \overline{3} \overline{4} \overline{6}]-[\overline{2} \overline{3} 46]-[23 \overline{4} \overline{6}]+[1 \overline{3} 46] \\
& +[\overline{1} 3 \overline{4} \overline{6}]+[234 \overline{5}]+[\overline{2} \overline{3} \overline{4} 5]+[\overline{1} 24 \overline{5}]+[1 \overline{2} \overline{4} 5]-[345 \overline{6}]-[\overline{3} \overline{4} \overline{5} 6]-[\overline{1} 35 \overline{6}]-[1 \overline{3} \overline{5} 6]-[12 \overline{3} 4]-[\overline{1} \overline{2} 3 \overline{4}] \\
& -[123 \overline{4}]-[\overline{1} \overline{2} \overline{3} 4]+[1 \overline{2} 3 \overline{4}]+[\overline{1} 2 \overline{3} 4]
\end{aligned}
$$

Now one single Plücker relation no longer suffices. The non-realizability follows from the existence of the following Plücker tree, which encodes a certain monomial combination of Plücker relations:

$$
\Gamma(124 \mid \overline{1} \overline{5} 6 \overline{6})-[1 \overline{1} 24 \overline{6}]^{?}-\Gamma(1 \overline{1} \overline{6} \mid 24 \overline{4} \overline{5})-[1 \overline{1} 4 \overline{4} \overline{6}]^{?}-\Gamma(\overline{1} \overline{4} \overline{6} \mid 1 \overline{2} 45)-[1 \overline{1} \overline{4} 5 \overline{6}]^{?}-\Gamma(1 \overline{4} \overline{6} \mid \overline{1} 2 \overline{2} 5)
$$

In this tree, which in this case is just a path, each edge is labeled by a determinant that occurs in both incident Plücker polynomials. In this example, it is also the case that the sign of these edge determinants (in red) is not known; but that doesn't matter, as they will be eliminated anyway. By successively eliminating the monomials containing these determinants (in any order, by Lemma 4.2), we arrive at a positive combination of monomials made from determinants that can be checked to be positive by the orientation above:

$$
\begin{aligned}
& {[12 \overline{6} \overline{4} \overline{2}]([\overline{1} \overline{2} \overline{6} \overline{4} \overline{5}]([1264 \overline{5}] \quad \Gamma(1 \overline{1} \overline{6} \mid 24 \overline{4} \overline{5})} \\
& +[1 \overline{4} \overline{\bar{c}} \overline{\mathrm{s}} \overline{1}](-\Gamma(124 \mid \overline{1} \overline{5} 6 \overline{6}))) \\
& +[12 \overline{5} \overline{6} \overline{1}][1264 \overline{5}](-\Gamma(\overline{1} \overline{4} \overline{6} \mid 1 \overline{2} 45))) \\
& +[12 \overline{5} \overline{6} \overline{1}][1264 \overline{5}][\overline{1} \overline{2} \overline{6} \overline{4} 4](-\Gamma(1 \overline{4} \overline{6} \mid \overline{1} 2 \overline{2} 5)) \\
& =[12 \overline{6} \overline{4} \overline{2}]\left([ \overline { 1 } \overline { 2 } \overline { 6 } \overline { 4 } 5 ] \left([1264 \overline{5}]\left([1 \overline{1} 24 \overline{6}]^{?}[1 \overline{4} \overline{6} \overline{\bar{L}} \overline{1}]+[12 \overline{6} \overline{4} \overline{1}][\overline{1} 4 \overline{6} \overline{5} 1]-[12 \overline{5} \overline{6} \overline{1}][1 \overline{1} 4 \overline{4} \overline{6}]^{?}\right)\right.\right. \\
& \left.+[1 \overline{4} \overline{6} \overline{5} \overline{1}]\left([\overline{1} 24 \overline{5} 1][1264 \overline{6}]+[1264 \overline{1}][12 \overline{5} \overline{6} 4]-[1 \overline{1} 24 \overline{6}]^{?}[1264 \overline{5}]\right)\right) \\
& \left.+[12 \overline{5} \overline{6} \overline{1}][1264 \overline{5}]\left([\overline{1} \overline{2} \overline{6} \overline{4} 1][\overline{1} 45 \overline{6} \overline{4}]+[1 \overline{1} 4 \overline{4} \overline{6}]^{?}[\overline{1} \overline{2} \overline{6} \overline{4} 5]+[1 \overline{1} \overline{4} 5 \overline{6}]^{?}[\overline{1} \overline{2} \overline{6} \overline{4} 4]\right)\right) \\
& +[12 \overline{5} \overline{6} \overline{1}][1264 \overline{5}][\overline{1} \overline{2} \overline{6} \overline{4} 4]\left([12 \overline{6} \overline{4} \overline{1}][1 \overline{2} \overline{4} 5 \overline{6}]+[\overline{1} \overline{2} \overline{6} \overline{4} 1][12 \overline{6} \overline{4} 5]-[1 \overline{1} \overline{4} 5 \overline{6}]^{?}[12 \overline{6} \overline{4} \overline{2} \overline{2}]\right) \\
& =[\overline{1} 24 \overline{5} 1][1 \overline{4} \overline{6} \overline{5} \overline{1}][12 \overline{6} \overline{4} \overline{2}][1264 \overline{6}][\overline{1} \overline{2} \overline{\bar{C}} \overline{4} 5]+[1264 \overline{1}][1 \overline{4} \overline{6} \overline{5} \overline{1}][12 \overline{6} \overline{4} \overline{2}][12 \overline{5} \overline{6} 4][\overline{1} \overline{2} \overline{\bar{C}} \overline{4} 5] \\
& +[12 \overline{6} \overline{4} \overline{1}][12 \overline{5} \overline{6} \overline{1}][1264 \overline{5}][1 \overline{2} \overline{4} 5 \overline{6}][\overline{1} \overline{2} \overline{6} \overline{4} 4]+[12 \overline{6} \overline{4} \overline{1}][\overline{1} 4 \overline{6} \overline{5} 1][12 \overline{6} \overline{4} \overline{2}][1264 \overline{5}][\overline{1} \overline{2} \overline{6} \overline{4} 5] \\
& +[12 \overline{5} \overline{6} \overline{1}][\overline{1} \overline{2} \overline{6} \overline{4} 1][12 \overline{6} \overline{4} \overline{2}][1264 \overline{5}][\overline{1} 45 \overline{6} \overline{4}]+[12 \overline{5} \overline{6} \overline{1}][\overline{1} \overline{2} \overline{6} \overline{4} 1][1264 \overline{5}][12 \overline{6} \overline{4} 5][\overline{1} \overline{2} \overline{6} \overline{4} 4] .
\end{aligned}
$$

The existence of this combination proves the non-realizability of Jockusch's sphere $\Delta_{6}^{3}$, because in any polytopal realization, all Plücker relations vanish, but all monomials in the final combination are positive. Moreover, the method detailed in Section 4.4 shows that this certificate is as short as possible.

Below, we build on this result and show that none of Jockusch's spheres $\Delta_{n}^{3}$ (Theorem 5.11), and indeed none of the spheres in Novik \& Zheng's family $\left\{\Delta_{n}^{d}: n-2 \geq d \geq 3\right\}$ (Theorem 5.13) are realizable. For now, after having seen the method at work in some examples, let's start again and carefully define all terms.

\section{Motivation AND OVERVieW}

Why do we even care about realizability? 
2.1. There are so many spheres. Which ones are actually polytopes? Write $s_{d}(n)$ for the number of combinatorial types of $d$-dimensional simplicial spheres on $n$ vertices, and $p_{d}(n)$ for the number of combinatorial types of $d$-dimensional simplicial polytopes on $n$ vertices. Goodman and Pollack [GP86], [Alo86] showed, suprisingly, that asymptotically there are only "very few" types of simplicial polytopes: in fixed $d$,

$$
p_{d}(n) \in 2^{\Theta_{d}(n \log n)} \text {. }
$$

Kalai [Kal88] used Stanley's Upper Bound Theorem [Sta75] for simplicial spheres and his own construction of "squeezed spheres" to establish astronomically larger upper and lower bounds for simplicial spheres:

$$
2^{\Omega\left(n^{\lfloor d / 2\rfloor}\right)} \leq s_{d}(n) \leq 2^{O\left(n^{\lceil d / 2\rceil} \log n\right)} .
$$

For even dimension $d$, these bounds already coincide up to a term of $\log n$ in the exponent. For $d=3$, the lower bound was first improved from $2^{\Omega(n)}$ to $2^{\Omega\left(n^{5 / 4}\right)}$ by Pfeifle and Ziegler [PZ04]. In general odd dimension $d=2 k-1$, the best result known to date is due to Nevo, Santos and Wilson [NSW16], who improved the lower bound from $2^{\Omega\left(n^{k-1}\right)}$ to $2^{\Omega\left(n^{k}\right)}$, asymptotically very close to the upper bound apart from, again, a factor of $\log n$ in the exponent.

As stated in the introduction, we suspect that deciding whether a simplicial complex $\Sigma$ that is guaranteed to be homeomorphic to a $(d-1)$-sphere is actually the boundary of a convex $d$-polytope is a very hard problem. Already for $d=4$ (in the non-simplicial case), it is equivalent to the "existential theory of the reals" [RZ95], which is known to be NP-hard by the results of Mnëv [Mne88] and Shor [Sho91]. According to Adiprasito and Padrol [AP17], the realization problem for neighborly spheres is "universal", i.e., for every "primary basic open semi-algebraic set over $\mathbb{Z}$ ", there exists a neighborly polytope with that realization space. But universality for simplicial polytopes in any fixed dimension $d$ remains open. Therefore, any new technique is welcome.

2.2. Plücker relations. A common strategy to prove non-realizability of a simplicial sphere $\Sigma$ is by exhibiting a property that $\Sigma$ should have if it were realizable, but showing that $\Sigma$ does not in fact have it.

One popular property of this kind is that realizable spheres should satisfy the Plücker relations. For this, suppose that $\Sigma$ is realized as the boundary complex of a simplicial convex polytope $P \subset \mathbb{R}^{d}$ with $n$ vertices, embed $P$ into the hyperplane $\left\{x=\left(x_{0}, x_{1}, \ldots, x_{d}\right) \in \mathbb{R}^{d+1}: x_{0}=1\right\}$, and write down the resulting $(d+1) \times n$ real matrix $V$ of homogeneous coordinates of $P$. The row space of $V$ corresponds to a point on the Grassmannian $\operatorname{Gr}(d+1, n)$. After embedding into $\mathbb{R} \mathbb{P}^{\left(\begin{array}{c}{[n]} \\ d+1\end{array}\right)}$, this point has Plücker coordinates ( $\operatorname{det} V_{J}: J \in\left(\begin{array}{c}{[n]} \\ d+1\end{array}\right)$ ), consisting of all $(d+1) \times(d+1)$-minors $V_{J}$ of $V$ enumerated in some fixed way, for example lexicographically. These coordinates satisfy the Plücker relations [BS89, Theorem 1.8], [MS15, Proposition 2.2.10], [MS05, Theorem 14.6]. In particular, any ordered $(d+3)$-tuple of indices $(S \mid i j k l)$ with $S \in\left(\begin{array}{c}{[n]} \\ d-1\end{array}\right)$ and $i, j, k, l \in[n] \backslash S$ gives rise to the 3 -term Plücker relation

$$
\Gamma(S \mid i j k l)=0
$$

for $\Sigma$, where

$$
\Gamma(S \mid i j k l):=[S i j][S k l]-[S i k][S j l]+[S i l][S j k] .
$$

We refer to [GMW20] for an overview of four different models of realization spaces of polytopes, which in particular relates this discussion to slack ideals and the slack variety [GMTW19] [BW19].

2.3. Overall strategy. Our strategy for proving the non-realizability of a simplicial complex $\Sigma$ by finding a final polynomial is analogous to the use of the Positivstellensatz [Stu02], [BS89, Chapter 4] to prove the non-existence of a solution to a system of real polynomial equations; see [DMP12] and e.g. [DId17].

Namely, we view the 3 -term Plücker relations as polynomials in the ring $\mathbb{R}[\boldsymbol{x}]:=\mathbb{R}\left[x_{J}: J \in\left(\begin{array}{c}{[n]} \\ d+1\end{array}\right)\right]$. We then find and exhibit a polynomial combination $\tau=\sum \kappa_{m} \Gamma_{m}$ of certain $\Gamma_{m}=\Gamma(S \mid i j k l)$ such that

- each coefficient $\kappa_{m} \in \mathbb{R}[\boldsymbol{x}]$ is a monomial that manifestly only takes on positive values for any value of $\boldsymbol{x}$ that comes from a realization of $\Sigma$;

- and the combination $\tau \in \mathbb{R}[\boldsymbol{x}]$ itself is manifestly a positive sum of monomials that also only take on positive values for any value of $\boldsymbol{x}$ that comes from a realization of $\Sigma$. 
The contradiction to realizability then arises from the fact that each $\Gamma_{m}$ vanishes for any value of $\boldsymbol{x}$ that comes from a realization of $\Sigma$; and we cannot get a positive value from a polynomial combination of zeroes.

We find these combinations by evaluating a certain tree whose nodes are Plücker polynomials; hence the name positive Plücker tree certificates.

\section{Technical apparatus}

3.1. Notation. Fix an integer $n \geq 3$. We set $[n]=\{1,2, \ldots, n\}$, and take the size of a set $S$ to be is its cardinality $|S|$. An (abstract) simplicial complex $\Sigma$ on $n$ vertices is a collection of subsets $\sigma \subset[n]$, called faces, such that the collection is closed under taking subsets. An inclusion-maximal face is called a facet. We will always assume that $\Sigma$ is pure, which means that all facets have the same cardinality. The dimension of a face $G$ of $\Sigma$ is $\operatorname{dim} G=|G|-1$, and the dimension of $\Sigma$ is the dimension of any facet. We set $d:=\operatorname{dim} \Sigma+1$, so that each facet of $\Sigma$ has cardinality $d$, and $n \geq d+1 \geq 3$. A ridge of $\Sigma$ is a face of dimension $(\operatorname{dim} \Sigma-1)$. The $k$-skeleton $\Sigma^{k}$ of $\Sigma$ is the set of its $k$-dimensional faces. The boundary $\partial \Sigma$ of $\Sigma$ is the set of ridges contained in exactly one facet.

Fix a $(k-1)$-dimensional face $\sigma \subset[n]$. Any permutation $\vec{\sigma}=\left[i_{1}, \ldots, i_{k}\right]$ of the vertices of $\sigma$ is called an oriented simplex. The support of $\vec{\sigma}$ is $\operatorname{supp} \vec{\sigma}=\left\{i_{1}, \ldots, i_{k}\right\}$. We will not obsess with notation, and happily confuse $\sigma$ with $\vec{\sigma}$ unless we fear misunderstanding.

A realization of $\Sigma$ in $\mathbb{R}^{d}$ is a set $X(\Sigma)=\left\{x_{1}, \ldots, x_{n}\right\}$ of points in $\mathbb{R}^{d}$ such that for each face $\sigma \in \Sigma$, the set conv $X_{\sigma}$ is a face of the convex hull conv $X$, where $X_{\sigma}:=\left\{x_{i}: i \in \sigma\right\}$. By this we mean the existence of an oriented hyperplane $H_{\sigma}=\left\{x \in \mathbb{R}^{d}:\left\langle a_{\sigma}, x\right\rangle=b_{\sigma}\right\} \subset \mathbb{R}^{d}$ such that conv $X_{\sigma} \subset H_{\sigma}$ and the remainder conv $X_{[n] \backslash \operatorname{supp} \sigma}$ lies strictly to one side of $H_{\sigma}$. We say that $\Sigma$ is non-realizable in $\mathbb{R}^{d}$ if no such assignment of points in $\mathbb{R}^{d}$ exists.

Since we attempt to realize the complex $\Sigma$ in $\mathbb{R}^{d}$, we are especially interested in the case $k=d+1$, which corresponds to full-dimensional simplices in $\mathbb{R}^{d}$. For this, we agree to use homogeneous coordinates for our points, so that $\operatorname{sign} \operatorname{det}\left(x_{i_{1}}, \ldots, x_{i_{d+1}}\right)$ is the orientation of a full-dimensional ordered simplex in $\mathbb{R}^{d}$.

We will need both the sign of the determinant $\operatorname{det} \sigma=\operatorname{det}\left(x_{i_{1}}, \ldots, x_{i_{d+1}}\right)$ determined by the permutation $\vec{\sigma}$, as well as the signature of the permutation $\vec{\sigma}=\left[i_{1}, \ldots, i_{d}\right]$ itself. We therefore adopt the mnemonic that $\nu(\sigma)$ is the sign of $\operatorname{det} \sigma$, and $\varepsilon(\sigma)=(-1)^{t(\vec{\sigma})}$ stands for the signature of $\vec{\sigma}$; here $t(\vec{\sigma})$ is the number of transpositions needed to build $\vec{\sigma}$ from the identity permutation. The two possible values \pm 1 of $\varepsilon(\sigma)$ correspond to the two equivalence classes of orientations of $\sigma$.

An oriented simplicial $k$-chain [Mun84] for $0 \leq k \leq \operatorname{dim} \Sigma$ is a formal sum $\sum_{\sigma \in \Sigma} c(\sigma) \vec{\sigma}$ of oriented $k$-dimensional simplices, with coefficients $c(\sigma) \in \mathbb{Z}$. We impose that $c(\vec{\sigma})=-c(-\vec{\sigma})$ whenever $-\vec{\sigma}$ is an oriented simplex of the opposite orientation as $\sigma$. The group of oriented simplicial $k$-chains is denoted $C_{k}(\Sigma)$. For $k<0$ or $k>\operatorname{dim} \Sigma$ we set $C_{k}(\Sigma)=0$. The boundary $\partial \vec{\sigma}$ of an oriented simplex is the image of $\vec{\sigma}$ under the map $\partial_{k}: C_{k}(\Sigma) \rightarrow C_{k-1}(\Sigma)$ given by

$$
\partial_{k} \vec{\sigma}=\partial\left[i_{0}, \ldots, i_{k}\right]=\sum_{j=0}^{k}(-1)^{j}\left[i_{0}, \ldots, \widehat{i_{j}}, \ldots, i_{k}\right] .
$$

The kernel of $\partial_{k}: C_{k}(\Sigma) \rightarrow C_{k-1}(\Sigma)$ is the group $Z_{k}(\Sigma)$ of $k$-cycles, while the image of $\partial_{k+1}:$ $C_{k+1}(\Sigma) \rightarrow C_{k}(\Sigma)$ is called the group $B_{k}(\Sigma)$ of $k$-boundaries. A standard calculation shows that $\partial_{k} \circ$ $\partial_{k+1}=0$, so that $B_{k}(\Sigma) \subset Z_{k}(\Sigma)$, i.e., every boundary of a $(k+1)$-chain is automatically a $k$-cycle. The $k$-th integral homology group of $\Sigma$ is $H_{k}(\Sigma ; \mathbb{Z}):=Z_{k}(\Sigma) / B_{k}(\Sigma)$.

A $(d-1)$-dimensional simplicial complex $\Sigma$ is

$\triangleright$ a closed pseudomanifold if each ridge is incident to exactly two facets;

$\triangleright$ orientable if it has the top-dimensional integral homology group of a sphere, $H_{d-1}(\Sigma ; \mathbb{Z}) \cong \mathbb{Z}$;

$\triangleright$ a homology sphere if all (reduced) homology groups coincide with those of a sphere, and the same holds true for all vertex links;

$\triangleright$ and a simplicial sphere if it is homeomorphic to a sphere.

Thus, any simplicial sphere is a pure, closed, orientable pseudomanifold and a homology sphere.

If $\Sigma$ is closed and orientable, an orientation of $\Sigma$ is a map $\omega: \Sigma^{d-1} \rightarrow\{+,-\}$ such that the simplicial chain $\sum_{\sigma \in \Sigma^{d-1}} \omega(\sigma) \vec{\sigma}$ generates $H_{d-1}(\Sigma ; \mathbb{Z})$. If $\Sigma$ is not closed, let $C_{1}, \ldots, C_{k}$ be the connected components of (the dual graph of) $\partial \Sigma$, and let $y_{1}, \ldots, y_{k}$ be new vertices. We extend $\Sigma$ to an enlarged complex $\widetilde{\Sigma}$ 
by coning over the boundary, i.e., adding all facets of the cones $y_{j} \star C_{j}$. In the case $H_{d-1}(\widetilde{\Sigma} ; \mathbb{Z})=\mathbb{Z}$, we define the orientation of $\Sigma$ to be the restriction of a generating cycle of $H_{d-1}(\widetilde{\Sigma} ; \mathbb{Z})$ to $\Sigma$, and we say that $\Sigma$ is an orientable pseudomanifold with boundary.

Convention 3.1. We will always assume that $\Sigma$ is an orientable, but not necessarily closed, pseudomanifold of dimension $\operatorname{dim} \Sigma=d-1$.

3.2. Nonrealizability certificates from Plïcker relations. Recall that our goal is to exploit positivity to prove that a $(d-1)$-dimensional simplicial complex $\Sigma$ given to us as a list of facets cannot be realized in convex position. Each 3-term Plücker relation

$$
\Gamma(S \mid i j k l)=[S i j][S k l]-[S i k][S j l]+[S i l][S j k]
$$

is a linear combination of products of determinants. If the signs of the determinants conspire together in such a way that all three terms in this combination have a positive coefficient (or all three have a negative coefficient), then the equation $\Gamma(S \mid i j k l)=0$ yields a contradiction to realizability: no sum of positive numbers (or of negative numbers) can be zero.

How can we control the signs of these determinants? Of course, we cannot control any of them individually, but in a convex realization we do know that many of them are equal. To see this, suppose we have a convex realization $X(\Sigma)$ of $\Sigma$, and consider any oriented facet $\vec{F}=\left(i_{1}, \ldots, i_{d}\right)$ of $\Sigma$. Because the realization is convex, all points $x_{j}$ with $j \notin \operatorname{supp} \vec{F}$ lie on the same side of the oriented hyperplane $H_{F}$. Another way of saying this is that the determinant $\operatorname{det}\left(x_{i_{1}}, \ldots, x_{i_{d}}, x_{j}\right)$ has the same sign for all indices $j \in[n] \backslash(\operatorname{supp} \vec{F})$, and this sign is determined by the orientation of $\vec{F}$.

To turn this dream into a working algorithm, we first need a normal form for our determinants.

Definition 3.2 (normal form). Fix an oriented, not necessarily closed pseudomanifold $\Sigma$ with orientation $\omega: \Sigma^{d-1} \rightarrow\{+,-\}$. An (oriented) solid in $\Sigma$ is an oriented simplex $\vec{\pi}=\left[i_{1}, \ldots, i_{d+1}\right]$ of size $d+1$. We say that an oriented solid $\vec{\pi}$ is known (i.e., we can control its orientation) via $F$ if there exists a facet $F \in \Sigma^{d-1}$ such that $F \subset \operatorname{supp} \vec{\pi}$, where $F$ is not necessarily uniquely determined. In this case, the facet $F$ is said to determine $\pi$, and the vertex of $\vec{\pi}$ associated to $F$ in $\pi$ is $\alpha(\pi, F)=(\operatorname{supp} \vec{\pi}) \backslash F$. For any oriented simplex $\sigma$, write $\sigma<$ for the reordering of $\sigma$ into ascending order.

The normal form $\bar{\pi}$ of $\vec{\pi}$ is defined as follows:

$\triangleright$ Suppose first that $\vec{\pi}$ is known via the facet $F \subset \Sigma^{d-1}$. Without loss of generality, suppose that $\vec{F}=$ $\vec{F}_{<}=\left(i_{1}, \ldots, i_{d}\right)$ with $i_{1}<\cdots<i_{d}$, and that the vertex of $\vec{\pi}$ associated to $F$ is $\alpha(\pi, F)=i_{d+1}$.

- If $\omega(\vec{F})=+$, then $\bar{\pi}=\left[i_{1}, \ldots, i_{d-2}, i_{d-1}, i_{d} \mid i_{d+1}\right]$ (see Convention 3.3).

- If $\omega(\vec{F})=-$, then $\bar{\pi}=\left[i_{1}, \ldots, i_{d-2}, i_{d}, i_{d-1} \mid i_{d+1}\right]$.

In particular, $\varepsilon(\bar{\pi})=\omega\left(\vec{F}_{<}\right) \varepsilon\left(\left[F_{<}, \alpha(\pi, F)\right]\right)$.

$\triangleright$ If $\vec{\pi}$ is not known, then $\bar{\pi}$ is the lexicographically ordered version of $\vec{\pi}: \bar{\pi}=\left[j_{1}, \ldots, j_{d+1}\right]$, where $j_{1}<\cdots<j_{d+1}$ and $\left\{j_{1}, \ldots, j_{d+1}\right\}=\operatorname{supp} \vec{\pi}$.

Convention 3.3. We sometimes typographically distinguish the normal form of a known solid $\pi$ (from that of an "unknown" solid) by means of the vertical bar "|" separating $F$ from $\alpha(\pi, F)$.

Sometimes, to emphasize that a solid $\bar{\pi}=\left[j_{1}, \ldots, j_{d+1}\right]$ is not known, we add a question mark as a superscript: $\bar{\pi}=\left[j_{1}, \ldots, j_{d+1}\right]^{\text {? }}$.

Proposition 3.4. (1) The signature $\varepsilon(\bar{\pi})$ of the normal form of an oriented solid $\vec{\pi}$ is well-defined, and $\varepsilon(\bar{\pi})=\omega\left(F_{<}\right) \varepsilon(\pi)$ if $\pi$ is known via $F$.

(2) If $\vec{\pi}$ is known with normal form $\bar{\pi}=\left[i_{1}, \ldots, i_{d} \mid i_{d+1}\right]$, we may assume withour loss of generality that $\nu(\bar{\pi})=\operatorname{sign} \operatorname{det}\left(x_{i_{1}}, \ldots, x_{i_{d+1}}\right)=+$ in any realization of $\Sigma$.

(3) For any oriented solid, $\pi=\varepsilon(\pi) \varepsilon(\bar{\pi}) \bar{\pi}$.

Before proving this, let us interject that if $\pi$ is known, then $\nu(\pi)=\varepsilon(\pi) \varepsilon(\bar{\pi}) \nu(\bar{\pi})=\varepsilon(\pi) \varepsilon(\bar{\pi})$ by Proposition 3.4 (2) and (3). However, if $\pi$ is not known, we have no control over $\nu(\pi)$. We formalize this state of affairs as follows.

Definition $3.5(\chi)$. If an oriented solid $\pi$ is known, set $\chi_{\pi}:=\chi(\pi):=\varepsilon(\pi) \varepsilon(\bar{\pi})$, otherwise set $\chi_{\pi}:=$ ? In all cases, we set $\chi_{\pi}^{2}=+$. 
We can therefore express the relationship between an oriented solid and its normal form as

$$
\pi=\chi_{\pi} \bar{\pi}
$$

Proof of Proposition 3.4. (1) For showing the well-definedness of $\varepsilon(\bar{\pi})$, we may suppose that $\operatorname{supp} \pi=$ $\{1,2, \ldots, d+1\}=F \cup\{k\}=G \cup\{\ell\}$, and write $R=F \cap G=\{1,2, \ldots, d+1\} \backslash\{k, \ell\}$ for the ridge common to $F$ and $G$. The two representations of $\pi$ are then as follows (suppose wlog $\ell<k$ ):

\begin{tabular}{c|cccc|cccc|ccc|c} 
position: & 1 & 2 & $\ldots$ & $\ell-1$ & $\ell$ & $\ell+1$ & $\ldots$ & $k-1$ & $k$ & $\ldots$ & $d$ & $d+1$ \\
\hline$F_{<} k:$ & 1 & 2 & $\ldots$ & $\ell-1$ & $\ell$ & $\ell+1$ & $\ldots$ & $k-1$ & $k+1$ & $\ldots$ & $d+1$ & $k$ \\
$G_{<} \ell:$ & 1 & 2 & $\ldots$ & $\ell-1$ & $\ell+1$ & $\ell+2$ & $\ldots$ & $k$ & $k+1$ & $\ldots$ & $d+1$ & $\ell$
\end{tabular}

We have written $F_{<}, G_{<}$to emphasize that $F$ and $G$ are sorted. By definition,

$$
\varepsilon\left(\overline{F_{<} k}\right)=\omega\left(F_{<}\right) \varepsilon\left(F_{<} k\right) \quad \text { and } \quad \varepsilon\left(\overline{G_{<\ell}}\right)=\omega\left(G_{<}\right) \varepsilon\left(G_{<} \ell\right),
$$

and we wish to show that these quantities are equal. Now

$$
\omega\left(F_{<}\right)=(-1)^{d-\ell} \omega\left(R_{<} \ell\right) \quad \text { and } \quad \omega\left(G_{<}\right)=(-1)^{d-k+1} \omega\left(R_{<} k\right),
$$

because to sort $F_{<}$into the form $R_{<} \ell$, we must swap the index $\ell$ to the right a total of $d-(\ell+1)+1$ steps. Similarly, to sort $G_{<}$into the form $R_{<} k$, we must swap the index $k$ to the right a total of $d-k+1$ steps. Next,

$$
\varepsilon\left(F_{<} k\right)=(-1)^{d-k+1} \quad \text { and } \quad \varepsilon\left(G_{<\ell}\right)=(-1)^{d-\ell+1},
$$

because to sort $F_{<} k$ we must bring $k$ to the left a total of $d-k+1$ steps, and to sort $G<\ell$ we must bring $\ell$ to the left a total of $d-\ell+1$ steps. Finally,

$$
\omega\left(R_{<} \ell\right)=-\omega\left(R_{<} k\right)
$$

because $\omega$ is an orientation. Substituing (3.2), (3.3) and (3.4) into (3.1) completes the proof.

(2) Since $\bar{\pi}$ is sorted such that the facet $F$ comes first, and $F$ indexes a facet of the convex hull in any realization of $\Sigma$, the $\operatorname{sign} \nu(\bar{\pi})$ is the same for all choices of $i_{d+1} \in[n] \backslash \operatorname{supp} F$. Moreover, since $\omega$ is an orientation, this sign is consistent across all facets $F$ of $\Sigma$. After a global reflection in $\mathbb{R}^{d}$, if necessary, we may therefore assume that $\nu(\bar{\pi})=+$.

(3) The sign $\varepsilon(\pi)$ reflects the change in signature as we permute $\pi$ to the identity, and $\varepsilon(\bar{\pi})$ accounts for permuting the identity into $\bar{\pi}$.

Observation 3.6. For any ordering $\sigma$ of $S$ and any permutation $\rho$ of the ordered sequence $(i, j, k, l)$, the sign of the Plücker relation only changes with the signature of $\rho$ :

$$
\Gamma(\sigma \mid \rho)=\varepsilon(\rho) \Gamma(S \mid i j k l) .
$$

Whether $\Gamma(S \mid \rho)=0$ or not is independent of $\epsilon(\rho)$, so there is no loss of generality in assuming that

$$
1 \leq i<j<k<l \leq n \text {. }
$$

\section{PlüCKer trees}

4.1. Combining two Plücker relations. When trying to prove non-realizability of a simplicial complex $\Sigma$, in general we won't be so lucky as to find a positive Plücker relation for $\Sigma$ as in Example 1.1. To find a sum of products of known-positive determinants that sums to zero, we need to combine several relations.

Suppose that two Plücker relations $\Gamma_{1}=\Gamma\left(\mathrm{S}_{1} \mid \mathrm{a}_{1}^{\prime} \mathrm{a}_{1}^{\prime \prime} \mathrm{b}^{\prime} \mathrm{b}^{\prime \prime}\right)$ and $\Gamma_{2}=\Gamma\left(\mathrm{S}_{2} \mid \mathrm{a}_{2}^{\prime} \mathrm{a}_{2}^{\prime \prime} \mathrm{c}^{\prime} \mathrm{c}^{\prime \prime}\right)$ share the same solid

$$
A=S_{1} \cup\left\{a_{1}^{\prime}, a_{1}^{\prime \prime}\right\}=S_{2} \cup\left\{a_{2}^{\prime}, a_{2}^{\prime \prime}\right\}
$$

possibly with different permutations of its elements. Written out and partially normalized, they read

$$
\begin{aligned}
& \Gamma_{1}=\Gamma\left(S_{1} \mid a_{1}^{\prime} a_{1}^{\prime \prime} b^{\prime} b^{\prime \prime}\right)=\quad\left[S_{1} a_{1}^{\prime} a_{1}^{\prime \prime}\right]\left[S_{1} b^{\prime} b^{\prime \prime}\right]-\left[S_{1} a_{1}^{\prime} b^{\prime}\right]\left[S_{1} a_{1}^{\prime \prime} b^{\prime \prime}\right]+\left[S_{1} a_{1}^{\prime} b^{\prime \prime}\right]\left[S_{1} a_{1}^{\prime \prime} b^{\prime}\right] \\
& =\chi([\mathrm{A}]) \chi\left(\left[\mathrm{S}_{1} \mathrm{~b}^{\prime} \mathrm{b}^{\prime \prime}\right]\right) \cdot[\overline{\mathrm{A}}]\left[\overline{\mathrm{S}_{1} \mathrm{~b}^{\prime} \mathrm{b}^{\prime \prime}}\right]-\left[\mathrm{S}_{1} \mathrm{a}_{1}^{\prime} \mathrm{b}^{\prime}\right]\left[\mathrm{S}_{1} \mathrm{a}_{1}^{\prime \prime} \mathrm{b}^{\prime \prime}\right]+\left[\mathrm{S}_{1} \mathrm{a}_{1}^{\prime} \mathrm{b}^{\prime \prime}\right]\left[\mathrm{S}_{1} \mathrm{a}_{1}^{\prime \prime} \mathrm{b}^{\prime}\right] \text {, } \\
& \Gamma_{2}=\Gamma\left(S_{2} \mid a_{2}^{\prime} a_{2}^{\prime \prime} c^{\prime} c^{\prime \prime}\right)=\quad\left[S_{2} a_{2}^{\prime} a_{2}^{\prime \prime}\right]\left[S_{2} c^{\prime} c^{\prime \prime}\right]-\left[S_{2} a_{2}^{\prime} c^{\prime}\right]\left[S_{2} a_{2}^{\prime \prime} c^{\prime \prime}\right]+\left[S_{2} a_{2}^{\prime} c^{\prime \prime}\right]\left[S_{2} a_{2}^{\prime \prime} c^{\prime}\right] \\
& \left.=\chi([\mathrm{A}]) \chi\left(\left[\mathrm{S}_{2} \mathrm{c}^{\prime} \mathrm{c}^{\prime \prime}\right]\right) \cdot[\overline{\mathrm{A}}] \overline{\left[\mathrm{S}_{2} \mathrm{c}^{\prime} \mathrm{c}^{\prime \prime}\right.}\right]-\left[\mathrm{S}_{2} \mathrm{a}_{2}^{\prime} \mathrm{c}^{\prime}\right]\left[\mathrm{S}_{2} \mathrm{a}_{2}^{\prime \prime} \mathrm{c}^{\prime \prime}\right]+\left[\mathrm{S}_{2} \mathrm{a}_{2}^{\prime} \mathrm{c}^{\prime \prime}\right]\left[\mathrm{S}_{2} \mathrm{a}_{2}^{\prime \prime} \mathrm{c}^{\prime}\right] \text {. }
\end{aligned}
$$


To eliminate the term containing A from them, we form the combination

$$
\begin{aligned}
&\left.\left.\overline{\left[\mathrm{S}_{2} \mathrm{c}^{\prime} \mathrm{c}^{\prime \prime}\right.}\right] \Gamma_{1}+s \overline{\left[\mathrm{S}_{1} \mathrm{~b}^{\prime} \mathrm{b}^{\prime \prime}\right.}\right] \Gamma_{2}=\left.\overline{\left[\mathrm{S}_{2} \mathrm{c}^{\prime} \mathrm{c}^{\prime \prime}\right.}\right]\left(-\left[\mathrm{S}_{1} \mathrm{a}_{1}^{\prime} \mathrm{b}^{\prime}\right]\left[\mathrm{S}_{1} \mathrm{a}_{1}^{\prime \prime} \mathrm{b}^{\prime \prime}\right]+\left[\mathrm{S}_{1} \mathrm{a}_{1}^{\prime} \mathrm{b}^{\prime \prime}\right]\left[\mathrm{S}_{1} \mathrm{a}_{1}^{\prime \prime} \mathrm{b}^{\prime}\right]\right) \\
&\left.+s \overline{\left[\mathrm{S}_{1} \mathrm{~b}^{\prime} \mathrm{b}^{\prime \prime}\right.}\right]\left(-\left[\mathrm{S}_{2} \mathrm{a}_{2}^{\prime} \mathrm{c}^{\prime}\right]\left[\mathrm{S}_{2} \mathrm{a}_{2}^{\prime \prime} \mathrm{c}^{\prime \prime}\right]+\left[\mathrm{S}_{2} \mathrm{a}_{2}^{\prime} \mathrm{c}^{\prime \prime}\right]\left[\mathrm{S}_{2} \mathrm{a}_{2}^{\prime \prime} \mathrm{c}^{\prime}\right]\right),
\end{aligned}
$$

where the elimination sign $s$ equals

$$
s=-\chi^{2}([\mathrm{~A}]) \chi\left(\left[\mathrm{S}_{1} \mathrm{~b}^{\prime} \mathrm{b}^{\prime \prime}\right]\right) \chi\left(\left[\mathrm{S}_{2} \mathrm{c}^{\prime} \mathrm{c}^{\prime \prime}\right]\right)=-\chi\left(\left[\mathrm{S}_{1} \mathrm{~b}^{\prime} \mathrm{b}^{\prime \prime}\right]\right) \chi\left(\left[\mathrm{S}_{2} \mathrm{c}^{\prime} \mathrm{c}^{\prime \prime}\right]\right) .
$$

In particular, the elimination sign does not depend on $[\mathrm{A}]$. We represent this elimination as follows:

$$
\Gamma\left(\mathrm{S}_{1} \mid \mathrm{a}_{1}^{\prime} \mathrm{a}_{1}^{\prime \prime} \mathrm{b}^{\prime} \mathrm{b}^{\prime \prime}\right)-[\mathrm{A}]-\Gamma\left(\mathrm{S}_{2} \mid \mathrm{a}_{2}^{\prime} \mathrm{a}_{2}^{\prime \prime} \mathrm{c}^{\prime} \mathrm{c}^{\prime \prime}\right)
$$

The case of most interest to us is when $\chi([\mathrm{A}])$ is unknown and $\chi\left(\left[\mathrm{S}_{1} \mathrm{~b}^{\prime} \mathrm{b}^{\prime \prime}\right]\right) \chi\left(\left[\mathrm{S}_{2} \mathrm{c}^{\prime} \mathrm{c}^{\prime \prime}\right]\right)=-1$, because then the elimination sign $s=1$, and the combination (4.1) contains one fewer solid of unknown sign.

\subsection{Combining eliminations into trees.}

Definition 4.1. Let $\Sigma$ be a simplicial complex on $n$ vertices of dimension $d-1$.

(1) The certificate ring $R[\Sigma]$ is the polynomial ring on the $\left(\begin{array}{c}n \\ d+1\end{array}\right)$ normal forms $\bar{\pi}$ of solids $\pi \in\left(\begin{array}{c}{[n]} \\ d+1\end{array}\right)$ with coefficients in some base ring $R$; for definiteness, we use $R=\mathbb{Z}$. In particular, Plücker relations of $\Sigma$ lie in $R[\Sigma]$.

(2) A Plücker tree $T$ for $\Sigma$ is a tree whose nodes are labeled with Plücker relations for $\Sigma$, and where each edge is labeled with a solid contained in both relations attached to the incident nodes. We will usually confuse nodes and edges with their labels. For any two nodes $\Gamma_{1}, \Gamma_{2}$ connected by an edge $[\mathrm{A}]$, we require that the monomial containing $[\mathrm{A}]$ have different sign in $\Gamma_{1}$ and $\Gamma_{2}$.

(3) Eliminating an edge in a Plücker tree consists of replacing an edge $[\mathrm{A}]$ and its incident nodes $\Gamma_{1}$, $\Gamma_{2}$ by a new node containing the combined relation according to (4.1), and connecting it to all neighbors of $\Gamma_{1}, \Gamma_{2}$. The labels of the new edges are assigned according to the connected nodes.

(4) The Plücker certificate $Z(T)$ obtained from $T$ is the polynomial in $R[\Sigma]$ contained in the only node left after all edges of $T$ have been eliminated.

(5) A Plücker tree is positive if $Z(T)$ is a sum of products of normal forms known to be positive (see Definition 3.2), with positive coefficients.

Obviously, the existence of a positive Plücker tree implies the non-realizability of $\Sigma$.

Lemma 4.2. The Plücker certificate $Z(T)$ does not depend on the order in which the edges are eliminated.

Proof. Root $T$ from any node by directing its edges away from that node, and consider any node $N$ of $T$. If $N$ is a leaf, there is nothing to prove. If $N$ has exactly one child and this child is a leaf, the corresponding edge may be eliminated uniquely, so again there is nothing to prove.

We may therefore suppose that $N$ has at least two children that are leaves. By induction, assume that $N$ is labeled by a polynomial $p \in R[\Sigma]$. Further assume that two of its incident leaf nodes are labeled by Plücker polynomials $\Gamma_{1}$ and $\Gamma_{2}$, and that the edges $\left\{p, \Gamma_{1}\right\}$ and $\left\{p, \Gamma_{2}\right\}$ are labeled by $[\mathrm{A}]$ and $[\mathrm{B}]$, respectively. Since the edge labeling is injective, we can moreover assume that

- $p=c_{0}[\mathrm{~A}]+d_{0}[\mathrm{~B}]+R_{0}$ for some polynomials $c_{0}, d_{0}, R_{0} \in R[\Sigma]$,

- $\Gamma_{1}=-c_{1}[\mathrm{~A}]+R_{1}$ for some $c_{1}, R_{1} \in R[\Sigma]$,

- $\Gamma_{2}=-d_{1}[\mathrm{~B}]+R_{2}$ for some $d_{1}, R_{2} \in R[\Sigma]$.

The proof now follows from Figure 1.

Remark 4.3. What we are doing is reminiscent of the approach in Corollary 3.13 in [BS89], except that we only use Plücker polynomials and not the van der Waerden identity, and we only use monomials and not binomials as coefficients.

4.3. The GP graph: where positive Plücker trees hide. Let us define the multigraph on the set of Plücker relations on which we will search for our Plücker tree certificates.

Definition 4.4 (Canonical signs; no two adjacent unknown signs). Let $\Gamma= \pm \Gamma(S \mid i j k l)$ be a Plücker relation or its negative. 


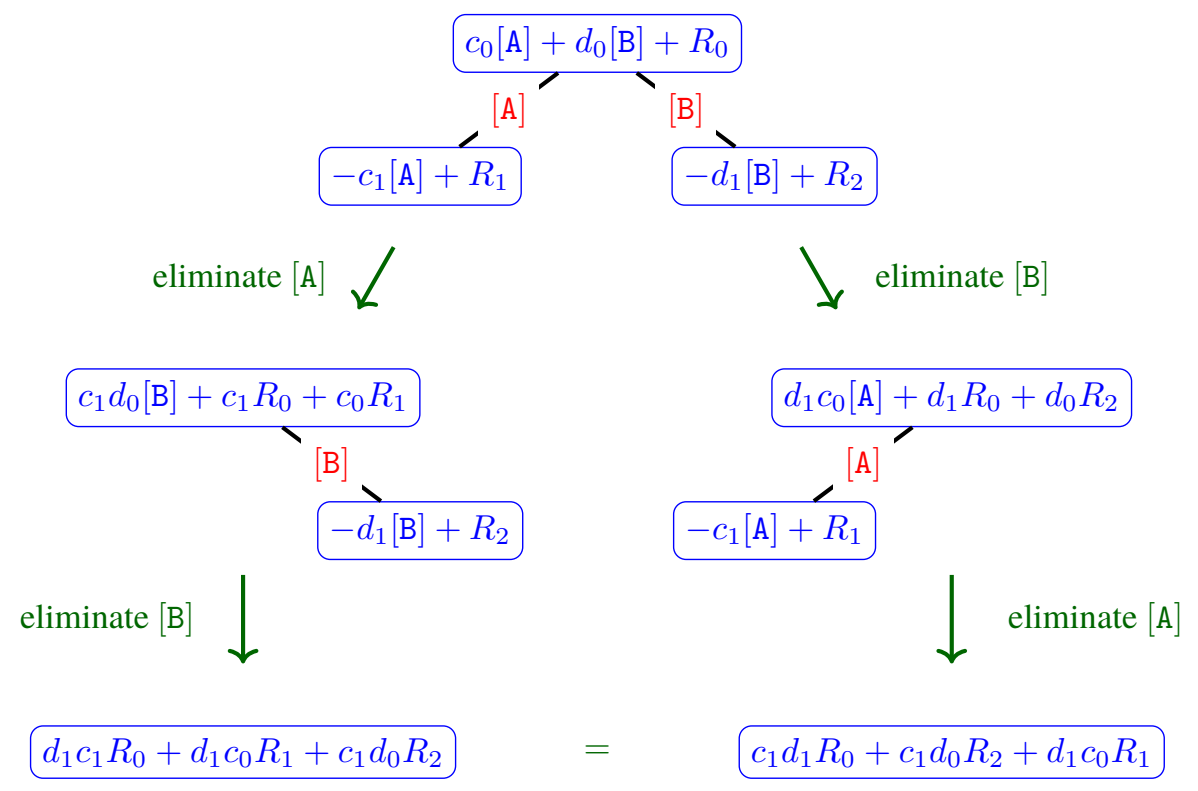

FIGURE 1. Eliminating edges in any order gives the same result

(1) The canonical signs $\sigma_{1}, \sigma_{2}, \sigma_{3} \in\{+,-, ?\}$ of $\Gamma$ are

$$
\sigma_{1}:= \pm \chi_{[S i j]} \chi_{[S k l]}, \quad \sigma_{2}:=\mp \chi_{[S i k]} \chi_{[S j l]}, \quad \sigma_{3}:= \pm \chi_{[S i l]} \chi_{[S j k]},
$$

so that

$$
\begin{aligned}
\pm \Gamma(S \mid i j k l) & = \pm[S i j][S k l] \mp[S i k][S j l] \pm[S i l][S j k] \\
& = \pm \chi_{[S i j]} \chi_{[S k l]} \overline{[S i j]} \overline{[S k l]} \mp \chi_{[S i k]} \chi_{[S j l]} \overline{[S i k]} \overline{[S j l]} \pm \chi_{[S i l]} \chi_{[S j k]} \overline{[S i l]} \overline{[S j k]} \\
& =: \sigma_{1} \overline{[S i j]} \overline{[S k l]}+\sigma_{2} \overline{[S i k]} \overline{[S j l]}+\sigma_{3} \overline{[S i l]} \overline{[S j k]} \\
& =: \sigma_{1} s_{11} s_{12}+\sigma_{2} s_{21} s_{22}+\sigma_{3} s_{31} s_{32} .
\end{aligned}
$$

Here we multiply unknown signs via the rules $(+)(?)=$ ? and $(-)(?)=?$. This is called a fuzzy ring in [BS89, Chapter 3].

(2) The relation $\Gamma$ has no two adjacent unknown solids if for each $i=1,2,3$, at least one of the signs of the solids corresponding to $\sigma_{i}$ is known.

Why do we not want a relation $\Gamma$ to have two adjacent unknown solids? Look at (4.1) and Figure 1: we use one of the solids as a coefficient for elimination, so it will most likely survive into the final certificate; therefore, we had better know its orientation.

Definition 4.5 (GP graph). Let $\Sigma$ be a simplicial complex, and let $\bar{S}_{\Sigma}$ be the set of canonical solids in $\Sigma$. The GP graph $\operatorname{GP}(\Sigma)=\left(V_{\Sigma}, E_{\Sigma}\right)$ of $\Sigma$ is an undirected graph on the node set

$$
V_{\Sigma}=\{ \pm \Gamma(S \mid i j k l): \Gamma(S \mid i j k \ell) \text { has no two adjacent unknown solids }\} .
$$

The edges of GP are colored with the set $\mathfrak{S}(\Sigma)$ of normal forms of solids of $\Sigma$. There can be multiple edges between two nodes, but they will have different colors. More precisely, two nodes $\Gamma, \Gamma^{\prime} \in V$ are joined with an edge $\left(\bar{\pi},\left\{\Gamma, \Gamma^{\prime}\right\}\right)$ colored $\bar{\pi} \in \mathfrak{S}(\Sigma)$ in $E_{\Sigma} \subseteq \mathfrak{S}(\Sigma) \times\left(\begin{array}{c}V_{\Sigma} \\ 2\end{array}\right)$ iff they share a solid $\pi$ such that

$$
\sigma_{i}=-\sigma_{i}^{\prime}
$$

where $\sigma_{i}, \sigma_{i}^{\prime}$ are the canonical signs of the terms containing $\pi$ in $\Gamma, \Gamma^{\prime}$.

Example 4.6 (Multiple edges in $\operatorname{GP}(\Sigma)$ ). Suppose that

$$
\begin{aligned}
& \Gamma(S \mid x j k l)=[S x j][S k l]-[S x k][S j l]+[S x l][S j k]=\overline{[S x j]} \overline{[S k l]}+\overline{[S x k]} \overline{[S j l]}+\overline{[S x l]} \overline{[S j k]}, \\
& \Gamma(S \mid y j k l)=[S y j][S k l]-[S y k][S j l]+[S y l][S j k]=-\overline{[S y j]} \overline{[S k l]}-\overline{[S y k]} \overline{[S j l]}-\overline{[S y l]} \overline{[S j k]}
\end{aligned}
$$


are nodes of $\operatorname{GP}(\Sigma)$. Then they are connected by three edges with colors $\overline{[S k l]}, \overline{[S j l]}$ and $\overline{[S j k]}$.

A Plücker tree $T$ is an induced subgraph of the GP graph such that

- for each unknown solid $\bar{\pi}^{\text {? }}$ (i.e., $\chi_{\pi}=$ ?) in a node $\Gamma$, there is an edge colored $\bar{\pi}$ incident to $\Gamma$;

- $T$ is a tree, i.e, $|V(T)|=|E(T)|+1$.

4.4. Using integer programming. We search for such a tree by solving the integer program on the integer indicator variables $\left\{x_{\Gamma}: \Gamma \in V_{\Sigma}\right\}$ and $\left\{x_{e}: e=\left(\bar{\pi},\left\{\Gamma, \Gamma^{\prime}\right\}\right) \in E_{\Sigma}\right\}$ defined as follows:

$$
\begin{aligned}
& \min \sum_{\Gamma \in V_{\Sigma}} x_{\Gamma} \\
& \text { s.t. } \quad \sum_{\bar{\pi}: e=\left(\bar{\pi},\left\{\Gamma, \Gamma^{\prime}\right\}\right) \in E_{\Sigma}} x_{e} \leq x_{\Gamma}+x_{\Gamma^{\prime}} \quad \text { for each }\left\{\Gamma, \Gamma^{\prime}\right\} \in\left(\begin{array}{c}
V_{\Sigma} \\
2
\end{array}\right) \\
& \sum_{\bar{\pi}: e=\left(\bar{\pi},\left\{\Gamma, \Gamma^{\prime}\right\}\right) \in E_{\Sigma}} x_{e} \leq 1 \quad \text { for each }\left\{\Gamma, \Gamma^{\prime}\right\} \in\left(\begin{array}{c}
V_{\Sigma} \\
2
\end{array}\right), \\
& 1+\sum_{e \in E_{\Sigma}} x_{e}=\sum_{\Gamma \in V_{\Sigma}} x_{\Gamma} \\
& \sum_{\Gamma^{\prime}: e=\left(\bar{\pi}^{?},\left\{\Gamma, \Gamma^{\prime}\right\}\right) \in E_{\Sigma}} x_{e}=x_{\Gamma} \quad \text { for all } \Gamma \in V_{\Sigma}, \text { for all unknown } \bar{\pi}^{?} \in \Gamma
\end{aligned}
$$

These inequalities for $x_{\Gamma}, x_{e} \in\{0,1\}$ have the following interpretation:

- (4.2) ensures that both endpoints of an edge present in the solution are present;

- (4.3) ensures that at most one edge is selected between two selected nodes;

- (4.4) forces the solution to be a tree with at least one node;

- (4.5) ensures that if a node $\Gamma$ with an unknown sign $\bar{\pi}^{\text {? }}$ is present in the solution, then there is exactly one edge of that color incident to $\Gamma$;

\section{Results}

We have implemented a search for positive Plücker trees in the software framework polymake [GJ00]. Obviously, there are many details of this implementation which we will not discuss in this paper, such as taking into account possible symmetries of the simplicial complex, using appropriate data structures, etc.

We remark that in all cases, the corresponding integer program was solved to optimality, proving the exhibited positive Plücker trees to be smallest possible.

5.1. Zheng's balanced 3-sphere. In [Zhe20a], Hailun Zheng constructs a combinatorial 3 -sphere $Z$ on 16 vertices with $f$-vector $(16,96,160,80)$ and an action of the dihedral group $D_{4}$ of order 8 . The 1 -skeleton of $Z$ is the complete multipartite graph $K_{4,4,4,4}$, which implies that it is balanced 2-neighborly.

To explain why this sphere is important, let's fix definitions. A $(d-1)$-dimensional simplicial complex is balanced if its 1-skeleton is $d$-colorable in the graph-theoretic sense, i.e., its vertices can be colored with $d$ colors in such a way that the endpoints of all edges receive different colors. Moreover, a $(d-1)$ dimensional balanced simplicial complex $\Sigma$ is balanced $k$-neighborly if each $k$-subset of the vertex set that contains at most one vertex of each color class is actually a face of $\Sigma$.

Now we can say why Zheng's example is important — in fact, it is important in at least two ways.

First, there has been a lot of work on analogies between combinatorial data in the balanced and the non-balanced settings [JM18, JMNS18, Ven19]. For example, one would like to have a balanced analogue of the celebrated Upper Bound Theorem by McMullen and Stanley. For this, in particular one would like balanced analogues of the extremal examples to even exist, i.e., one would like to construct infinite families of balanced $k$-neighborly simplicial spheres. What Zheng shows in [Zhe20a], however, is that (i) there is no balanced 2-neighborly homology 3-sphere on 12 vertices; (ii) there is no balanced 2-neighborly homology 4-sphere on 15 vertices; (iii) but taking suspensions over her example $Z$ yields a balanced 2-neighborly homology $(3+m)$-sphere on $16+2 m$ vertices for every $m \geq 0$.

The second reason why her example is important lies in the fact that in [PPS12], the present author, Vincent Pilaud and Francisco Santos study which graphs are realizable as the 1-skeleton of polytopes. The 
case of multipartite graphs was not treated there, and to date the only polytope whose graph is known to be the multipartite graph $K_{4,4,4,4}$ is the 4-dimensional cross polytope.

We can now show for the first time that $Z$ is not realizable as the boundary complex of a convex polytope, and therefore that $Z$ does not yield a new polytope whose graph is $K_{4,4,4,4}$.

Theorem 5.1. Zheng's balanced sphere $Z$ is not polytopal.

Proof. We enumerate and orient the facets of $Z$ :

$-[048 c]+[048 \mathrm{e}]+[049 \mathrm{c}]-[049 \mathrm{~d}]+[04 \mathrm{ad}]-[04 \mathrm{ae}]+[059 \mathrm{~d}]-[059 f]-[05 \mathrm{ad}]+[05 \mathrm{ae}]$
$-[05 \mathrm{be}]+[05 \mathrm{bf}]+[068 \mathrm{c}]-[068 \mathrm{e}]-[069 \mathrm{c}]+[069 \mathrm{e}]-[079 \mathrm{e}]+[079 f]+[07 \mathrm{be}]-[07 \mathrm{bf}]$
$+[148 \mathrm{c}]-[148 \mathrm{e}]+[14 \mathrm{ae}]-[14 \mathrm{af}]-[14 \mathrm{bc}]+[14 \mathrm{bf}]-[158 \mathrm{c}]+[158 \mathrm{~d}]-[159 \mathrm{~d}]+[159 f]$
$+[15 \mathrm{bc}]-[15 \mathrm{bf}]+[168 \mathrm{e}]-[168 \mathrm{f}]-[16 \mathrm{ae}]+[16 \mathrm{af}]-[178 \mathrm{~d}]+[178 \mathrm{f}]+[179 \mathrm{~d}]-[179 f]$
$-[24 \mathrm{ad}]+[24 \mathrm{af}]+[24 \mathrm{bd}]-[24 \mathrm{bf}]+[258 \mathrm{c}]-[258 \mathrm{~d}]-[25 \mathrm{ac}]+[25 \mathrm{ad}]-[268 \mathrm{c}]+[268 \mathrm{~d}]$
$+[269 \mathrm{c}]-[269 \mathrm{e}]+[26 \mathrm{ae}]-[26 \mathrm{af}]-[26 \mathrm{bd}]+[26 \mathrm{bf}]-[279 \mathrm{c}]+[279 \mathrm{e}]+[27 \mathrm{ac}]-[27 \mathrm{ae}]$
$-[349 \mathrm{c}]+[349 \mathrm{~d}]+[34 \mathrm{bc}]-[34 \mathrm{bd}]+[35 \mathrm{ac}]-[35 \mathrm{ae}]-[35 \mathrm{bc}]+[35 \mathrm{be}]-[368 \mathrm{~d}]+[368 f]$
$+[36 \mathrm{bd}]-[36 \mathrm{bf}+[378 \mathrm{~d}]-[378 \mathrm{f}]+[379 \mathrm{c}]-[379 \mathrm{~d}]-[37 \mathrm{ac}]+[37 \mathrm{ae}]-[37 \mathrm{be}]+[37 \mathrm{bf}]$

The non-realizability follows from the existence of the following Plücker tree:

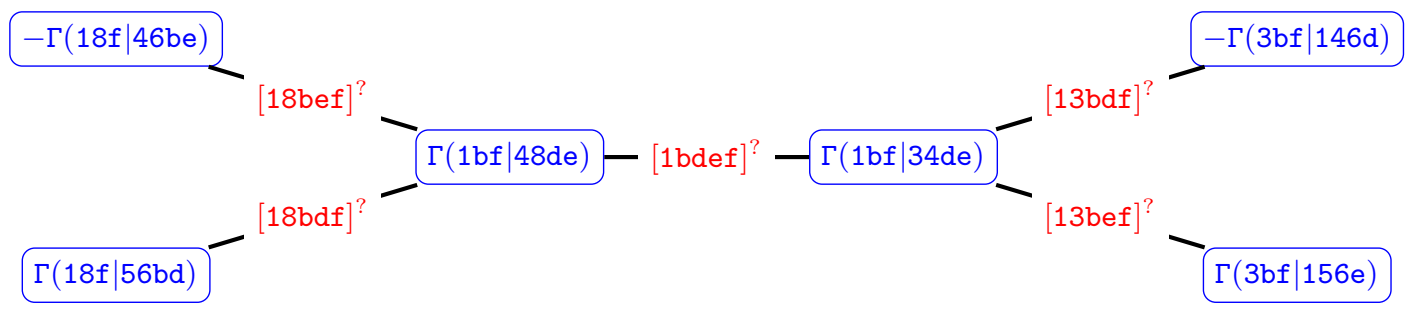

which gives rise to the following certificate, which is short enough to write down in full:

$$
\begin{array}{r}
{[36 \mathrm{fb} 5]([36 \mathrm{fb} 4]([14 \mathrm{bf} 3]([16 \mathrm{f} 85]([14 \mathrm{bfd}](-\Gamma(18 \mathrm{f} \mid 46 \mathrm{be}))} \\
+[16 \mathrm{f} 84] \Gamma(1 \mathrm{bf} \mid 48 \mathrm{de})) \\
+[16 \mathrm{f} 84][14 \mathrm{bfe}] \Gamma(18 \mathrm{f} \mid 56 \mathrm{bd})) \\
+[16 \mathrm{f} 84][14 \mathrm{bf} 8][16 \mathrm{f} 85] \Gamma(1 \mathrm{bf} \mid 34 \mathrm{de})) \\
+[16 \mathrm{f} 84][14 \mathrm{bf} 8][14 \mathrm{bfe}][16 \mathrm{f} 85](-\Gamma(3 \mathrm{bf} \mid 146 \mathrm{~d}))) \\
+[16 \mathrm{f} 84][14 \mathrm{bf} 8][14 \mathrm{bfd}][16 \mathrm{f} 85][36 \mathrm{fb} 4] \Gamma(3 \mathrm{bf} \mid 156 \mathrm{e})
\end{array} .
$$

Substituting the appropriately normalized Plücker polynomials yields

$$
\begin{array}{r}
{[36 \mathrm{fb} 5]\left([ 3 6 \mathrm { fb } 4 ] \left([ 1 4 \mathrm { bf } 3 ] \left([ 1 6 \mathrm { f } 8 5 ] \left([14 \mathrm{bfd}]\left(-[16 \mathrm{f} 84][18 \mathrm{bef}]^{?}+[14 \mathrm{bf} 8][16 \mathrm{f} 8 \mathrm{e}]+[14 \mathrm{e} 8 \mathrm{f}][16 \mathrm{ffb}]\right)\right.\right.\right.\right.} \\
\left.+[16 \mathrm{f} 84]\left([14 \mathrm{bf} 8][1 \mathrm{bdef}]^{?}+[14 \mathrm{bfd}][18 \mathrm{bef}]^{?}-[14 \mathrm{bfe}][18 \mathrm{bdf}]^{?}\right)\right) \\
\left.+[16 \mathrm{f} 84][14 \mathrm{bfe}]\left([16 \mathrm{f} 85][18 \mathrm{bdf}]^{?}+[15 \mathrm{fb} 8][16 \mathrm{f} 8 \mathrm{~d}]+[158 \mathrm{df}][16 \mathrm{f} 8 \mathrm{~b}]\right)\right) \\
\left.+[16 \mathrm{ff} 84][14 \mathrm{bf} 8][16 \mathrm{ff} 85]\left(-[14 \mathrm{bf} 3][1 \mathrm{bdef}]^{?}+[13 \mathrm{bdf}]^{?}[14 \mathrm{bfe}]-[13 \mathrm{bef}]^{?}[14 \mathrm{bfd}]\right)\right) \\
\left.+[16 \mathrm{f} 84][14 \mathrm{bf} 8][14 \mathrm{bfe}][16 \mathrm{f} 85]\left([14 \mathrm{bf} 3][36 \mathrm{fbd}]+[36 \mathrm{fb} 1][34 \mathrm{dbf}]-[13 \mathrm{bdf}]^{?}[36 \mathrm{fb} 4]\right)\right) \\
+[16 \mathrm{f} 84][14 \mathrm{bf} 8][14 \mathrm{bfd}][16 \mathrm{f} 85][36 \mathrm{fb} 4]\left([15 \mathrm{fb} 3][36 \mathrm{fbe}]+[36 \mathrm{fb} 1][35 \mathrm{bef}]+[13 \mathrm{bef}]^{?}[36 \mathrm{fb} 5]\right)
\end{array}
$$


and this in turn simplifies to the final form of the certificate,

$$
\begin{aligned}
& {[14 \mathrm{bf} 3][16 \mathrm{f} 84][14 \mathrm{bf} 8][14 \mathrm{bfe}][16 \mathrm{f} 85][36 \mathrm{fb} 5][36 \mathrm{fbd}]+[14 \mathrm{bf} 3][16 \mathrm{f} 84][14 \mathrm{bfe}][15 \mathrm{fb} 8][16 \mathrm{f} 8 \mathrm{~d}][36 \mathrm{fb} 4][36 \mathrm{fb} 5] } \\
+ & {[14 \mathrm{bf} 3][16 \mathrm{f} 84][14 \mathrm{bfe}][158 \mathrm{df}][16 \mathrm{f} 8 \mathrm{~b}][36 \mathrm{fb} 4][36 \mathrm{fb} 5]+[14 \mathrm{bf} 3][14 \mathrm{bf} 8][14 \mathrm{bfd}][16 \mathrm{f} 85][16 \mathrm{f} 8 \mathrm{e}][36 \mathrm{fb} 4][36 \mathrm{fb} 5] } \\
+ & {[14 \mathrm{bf} 3][14 \mathrm{ef}][14 \mathrm{bfd}][16 \mathrm{f} 85][16 \mathrm{f} 8 \mathrm{~b}][36 \mathrm{fb} 4][36 \mathrm{fb} 5]+[15 \mathrm{fb} 3][16 \mathrm{f} 84][14 \mathrm{bf} 8][14 \mathrm{bfd}][16 \mathrm{f} 85][36 \mathrm{fb} 4][36 \mathrm{fbe}] } \\
+ & {[36 \mathrm{fb} 1][16 \mathrm{f} 84][14 \mathrm{bf} 8][14 \mathrm{bfd}][16 \mathrm{f} 85][36 \mathrm{fb} 4][35 \mathrm{bef}]+[36 \mathrm{fb} 1][16 \mathrm{f} 84][14 \mathrm{bf} 8][14 \mathrm{bfe}][16 \mathrm{f} 85][34 \mathrm{dbf}][36 \mathrm{fb} 5] }
\end{aligned}
$$

5.2. Topological Prismatoids. In [CS19], Francisco Criado and Francisco Santos introduced topological prismatoids, a combinatorial abstraction of the geometric prismatoids used by Santos [San12] to construct counterexamples to the Hirsch conjecture. Criado and Santos construct four combinatorially distinct non- $d$ step topological 4-dimensional prismatoids on 14 vertices, referred to as \#1039, \#1963, \#2669 and \#3513, which imply the existence of 8-dimensional spheres on 18 vertices whose combinatorial diameter exceeds the Hirsch bound. In [CS19], the question of polytopality of these combinatorial prismatoids was left open. Using our technique, we can prove that all four of them are in fact not polytopal.

Remark 5.2. During the elaboration of this manuscript, Gouveia, Macchia and Wiebe (see version v3 of [GMW20]) were also able to prove the non-realizability of the prismatoid \#1039; they will make nonrealizability proofs of the other ones available in a future paper [Wie20].

Remark 5.3. A technical detail worth mentioning is that each of these combinatorial prismatoids $\Pi$ has two non-simplicial facets. To construct an orientation of the boundary of $\Pi$, we first consider the simplicial sphere $\widetilde{\Pi}$ obtained by replacing each non-simplicial facet by a cone over its (simplicial) boundary. Next, we calculate a representative cycle for the top simplicial homology group of $\widetilde{\Pi}$, and restrict that cycle to the simplicial facets of $\Pi$.

\section{Theorem 5.4. The prismatoid \#1039 is not realizable.}

Proof. One of the two possible orientations of the simplicial facets of \#1039 is as follows. The nine facets relevant to the certificate are listed first:

$$
\begin{aligned}
& -[0145 f]+[014 \mathrm{ad}]-[014 \mathrm{ae}]-[014 \mathrm{bc}]+[014 \mathrm{bf}]-[014 \mathrm{~cd}]-[04 \mathrm{bcd}]-[05 \mathrm{bde}]+[05 \mathrm{cde}] \\
& -[0123 \mathrm{~d}]+[0126 \mathrm{~d}]-[0134 \mathrm{e}]-[013 \mathrm{ad}]+[013 \mathrm{ae}]-[0156 \mathrm{~g}]-[015 \mathrm{bf}]+[015 \mathrm{bg}]+[016 \mathrm{~cd}] \\
& -[016 \mathrm{cg}]+[01 \mathrm{bcg}]+[0234 \mathrm{e}]+[023 \mathrm{~cd}]-[023 \mathrm{ce}]+[0245 \mathrm{f}]+[024 \mathrm{ae}]-[024 \mathrm{af}]+[0256 \mathrm{~g}] \\
& -[025 \mathrm{ae}]+[025 \mathrm{af}]+[025 \mathrm{be}]-[025 \mathrm{bg}]-[026 \mathrm{be}]+[026 \mathrm{bg}]-[026 \mathrm{~cd}]+[026 \mathrm{ce}]+[03 \mathrm{acd}] \\
& -[03 \mathrm{ace}]-[04 \mathrm{abd}]+[04 \mathrm{abf}]+[05 \mathrm{abd}]-[05 \mathrm{abf}]-[05 \mathrm{acd}]+[05 \mathrm{ace}]+[06 \mathrm{bce}]-[06 \mathrm{bcg}] \\
& -[0 \mathrm{bcde}]-[1234 \mathrm{e}]+[123 \mathrm{ae}]-[123 \mathrm{af}]+[123 \mathrm{bf}]-[123 \mathrm{bg}]-[123 \mathrm{~cd}]+[123 \mathrm{cg}]-[1245 \mathrm{f}] \\
& -[124 \mathrm{ae}]+[124 \mathrm{af}]-[1256 \mathrm{~g}]-[125 \mathrm{bf}]+[125 \mathrm{bg}]+[126 \mathrm{~cd}]-[126 \mathrm{cg}]+[13 \mathrm{abf}]-[13 \mathrm{abg}] \\
& -[13 \mathrm{acd}]+[13 \mathrm{acg}]-[14 \mathrm{abf}]+[14 \mathrm{abg}]+[14 \mathrm{acd}]-[14 \mathrm{acg}]+[14 \mathrm{bcg}]+[23 \mathrm{ace}]-[23 \mathrm{acf}] \\
& +[23 \mathrm{bcf}]-[23 \mathrm{bcg}]-[25 \mathrm{abe}]+[25 \mathrm{abf}]+[26 \mathrm{abe}]-[26 \mathrm{abf}]-[26 \mathrm{ace}]+[26 \mathrm{acf}]-[26 \mathrm{bcf}] \\
& +[26 \mathrm{bcg}]-[3 \mathrm{abfg}]+[3 \mathrm{acfg}]-[3 \mathrm{bcfg}]-[4 \mathrm{abcd}]+[4 \mathrm{abcg}]-[5 \mathrm{abde}]+[5 \mathrm{acde}]-[6 \mathrm{abef}] \\
& +[6 \mathrm{acef}]-[6 \mathrm{bcef}]
\end{aligned}
$$

The non-realizability follows from the existence of the following positive Plücker tree:

$$
\Gamma(0145 \mid \text { adef })-[0145 \mathrm{de}]^{?}-\Gamma(045 \mathrm{~d} \mid 1 \mathrm{bce})-[0145 \mathrm{bd}]^{?}-\Gamma(014 \mathrm{~d} \mid 5 \mathrm{bcf})
$$

which gives rise to the certificate

$$
\begin{aligned}
{[014 \mathrm{ad} 5][014 \mathrm{f} 5 \mathrm{e}][014 \mathrm{dcf}][04 \mathrm{bdc5}] } & +[014 \mathrm{ea} 5][014 \mathrm{f} 5 \mathrm{~d}][014 \mathrm{dcf}][04 \mathrm{bdc5}] \\
+[014 \mathrm{f} 5 \mathrm{a}][014 \mathrm{dc5}][014 \mathrm{bfd}][05 \mathrm{cde} 4] & +[014 \mathrm{f} 5 \mathrm{a}][014 \mathrm{dc5}][014 \mathrm{dcf}][05 \mathrm{bed} 4] \\
& +[014 \mathrm{f} 5 \mathrm{a}][014 \mathrm{f} 5 \mathrm{~d}][04 \mathrm{bdc1}][05 \mathrm{cde} 4]
\end{aligned}
$$

Theorem 5.5. The prismatoid \#1963 is not realizable.

Proof. The oriented simplicial facets are as follows, relevant ones first: 


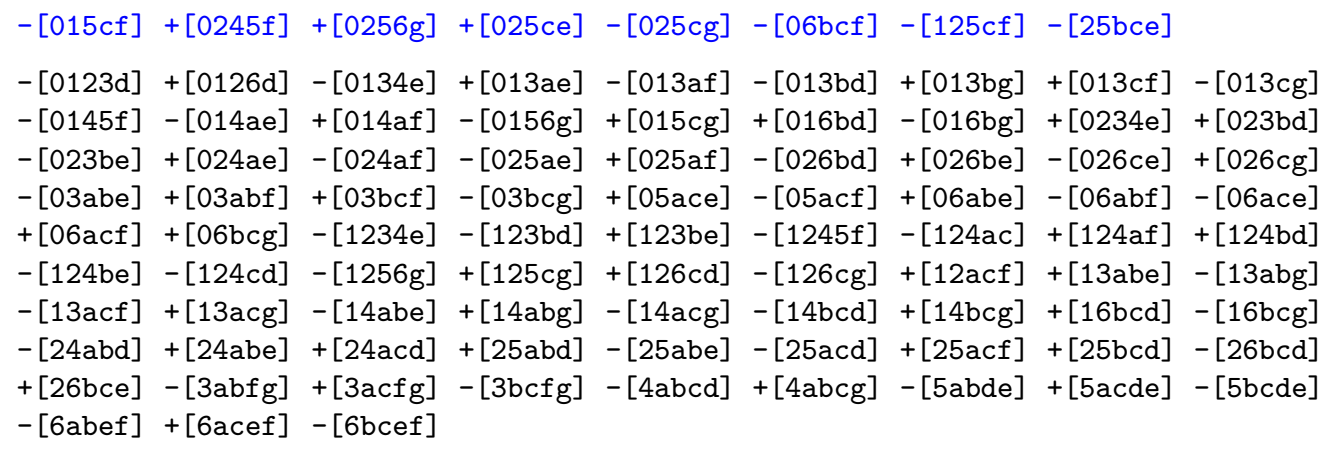

The non-realizability follows from the existence of the following positive Plücker tree:

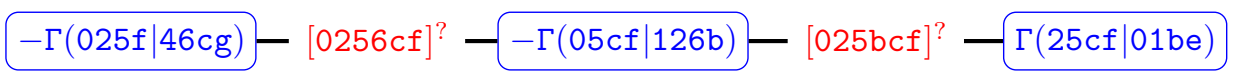

with final certificate

$$
\begin{array}{r}
{[125 \mathrm{fc} 0][015 \mathrm{fc} 6][0245 \mathrm{fg}][25 \mathrm{becf}]+[125 \mathrm{fc} 0][0245 \mathrm{fg}][06 \mathrm{bfc5}][125 \mathrm{fce}]} \\
+[015 \mathrm{fc} 6][0245 \mathrm{fg}][025 \mathrm{cef}][125 \mathrm{fcb}]+[015 \mathrm{fcb}][0245 \mathrm{f} 6][025 \mathrm{gcf}][125 \mathrm{fce}] \\
+[015 \mathrm{fcb}][0245 \mathrm{fc}][0256 \mathrm{gf}][125 \mathrm{fce}]
\end{array}
$$

Theorem 5.6. The prismatoid \#2669 is not realizable.

Proof. The oriented simplicial facets of \#2669 are (relevant ones first)

$$
\begin{aligned}
& +[0234 a]+[023 a d]+[123 a e]-[123 c d]-[13 a c d]-[14 a b d]+[14 a c d]-[234 a e]+[23 a b f] \\
& +[23 a c d]-[24 a b f]-[4 a b c d] \\
& -[0123 d]+[0126 \mathrm{~d}]-[0134 \mathrm{a}]-[013 \mathrm{ad}]+[013 \mathrm{ae}]-[0145 f]-[014 \mathrm{be}]+[014 \mathrm{bf}]-[0156 \mathrm{f}] \\
& +[015 \mathrm{ad}]-[015 \mathrm{ae}]+[015 \mathrm{be}]-[015 \mathrm{bf}]-[015 \mathrm{~cd}]+[015 \mathrm{cg}]+[016 \mathrm{~cd}]-[016 \mathrm{cg}]+[0245 \mathrm{f}] \\
& -[024 \mathrm{af}]+[0256 \mathrm{~g}]+[025 \mathrm{bf}]-[025 \mathrm{bg}]-[026 \mathrm{ad}]+[026 \mathrm{af}]-[026 \mathrm{bf}]+[026 \mathrm{bg}]+[034 \mathrm{ae}] \\
& -[04 \mathrm{abe}]+[04 \mathrm{abf}]-[05 \mathrm{acd}]+[05 \mathrm{ace}]-[05 \mathrm{bce}]+[05 \mathrm{bcg}]+[06 \mathrm{abe}]-[06 \mathrm{abf}]+[06 \mathrm{acd}] \\
& -[06 \mathrm{ace}]+[06 \mathrm{bce}]-[06 \mathrm{bcg}]-[1234 \mathrm{e}]-[123 \mathrm{ag}]+[123 \mathrm{cg}]-[1245 \mathrm{f}]-[124 \mathrm{ae}]+[124 \mathrm{ag}] \\
& +[124 \mathrm{bf}]-[124 \mathrm{bg}]-[1256 \mathrm{~g}]-[125 \mathrm{bf}]+[125 \mathrm{bg}]+[126 \mathrm{~cd}]-[126 \mathrm{cg}]+[13 \mathrm{acg}]+[14 \mathrm{abe}] \\
& -[14 \mathrm{acg}]-[14 \mathrm{bcd}]+[14 \mathrm{bcg}]+[15 \mathrm{abd}]-[15 \mathrm{abe}]+[15 \mathrm{bcd}]-[15 \mathrm{bcg}]-[23 \mathrm{abg}]-[23 \mathrm{acf}] \\
& +[23 \mathrm{bcf}]-[23 \mathrm{bcg}]+[24 \mathrm{abg}]-[26 \mathrm{acd}]+[26 \mathrm{acf}]-[26 \mathrm{bcf}]+[26 \mathrm{bcg}]-[3 \mathrm{abfg}]+[3 \mathrm{acfg}] \\
& -[3 \mathrm{bcfg}]+[4 \mathrm{abcg}]-[5 \mathrm{abde}]+[5 \mathrm{acde}]-[5 \mathrm{bcde}]-[6 \mathrm{abef}]+[6 \mathrm{acef}]-[6 \mathrm{bcef}]
\end{aligned}
$$

The non-realizability follows from the existence of the following positive Plücker tree:

$$
-\Gamma(23 \mathrm{ab} \mid 04 \mathrm{df})-[234 \mathrm{abd}]^{?}-\Gamma(24 \mathrm{ad} \mid 13 \mathrm{bc})-[1234 \mathrm{ad}]^{?}--\Gamma(23 \mathrm{ad} \mid 04 \mathrm{df})
$$

with final certificate

$$
\begin{aligned}
{[0234 \mathrm{ab}][14 \mathrm{acd} 2][23 \mathrm{abfd}][23 \mathrm{acde}] } & +[023 \mathrm{adb}][14 \mathrm{acd} 2][24 \mathrm{afb} 3][23 \mathrm{acde}] \\
+[23 \mathrm{abf0}][23 \mathrm{acd} 1][234 \mathrm{ead}][4 \mathrm{abdc} 2] & +[23 \mathrm{abf} 0][123 \mathrm{aed}][23 \mathrm{acd} 4][4 \mathrm{abdc} 2] \\
& +[23 \mathrm{abf0}][14 \mathrm{adb} 2][23 \mathrm{acd} 4][23 \mathrm{acde}] .
\end{aligned}
$$

Theorem 5.7. The prismatoid \#3513 is not realizable.

Proof. The oriented simplicial facets are (relevant ones first)

$$
\begin{aligned}
& +[014 \mathrm{af}]-[014 \mathrm{ag}]+[014 \mathrm{bg}]+[04 \mathrm{abg}]+[124 \mathrm{af}]+[125 \mathrm{bg}]-[13 \mathrm{abg}]-[14 \mathrm{acg}]+[14 \mathrm{bcg}] \\
& +[15 \mathrm{abg}]-[3 \mathrm{abfg}]+[4 \mathrm{abcg}]
\end{aligned}
$$




$$
\begin{aligned}
& -[0123 \mathrm{~d}]+[0126 \mathrm{~d}]-[0134 \mathrm{e}]-[013 \mathrm{~cd}]+[013 \mathrm{ce}]-[0145 \mathrm{f}]-[014 \mathrm{bc}]-[014 \mathrm{ce}]-[0156 \mathrm{~g}] \\
& -[015 \mathrm{af}]+[015 \mathrm{ag}]+[016 \mathrm{bd}]-[016 \mathrm{bg}]+[01 \mathrm{bcd}]+[0234 \mathrm{e}]+[023 \mathrm{~cd}]-[023 \mathrm{ce}]+[0245 \mathrm{f}] \\
& +[024 \mathrm{ae}]-[024 \mathrm{af}]+[0256 \mathrm{~g}]-[025 \mathrm{ae}]+[025 \mathrm{af}]+[025 \mathrm{bd}]-[025 \mathrm{bg}]-[025 \mathrm{~cd}]+[025 \mathrm{ce}] \\
& -[026 \mathrm{bd}]+[026 \mathrm{bg}]-[04 \mathrm{abd}]+[04 \mathrm{acd}]-[04 \mathrm{ace}]-[04 \mathrm{bcd}]+[05 \mathrm{abd}]-[05 \mathrm{abg}]-[05 \mathrm{acd}] \\
& +[05 \mathrm{ace}]-[1234 \mathrm{e}]+[123 \mathrm{ae}]-[123 \mathrm{af}]-[123 \mathrm{~cd}]+[123 \mathrm{cf}]-[1245 \mathrm{f}]-[124 \mathrm{ae}]-[1256 \mathrm{~g}] \\
& -[125 \mathrm{bf}]+[126 \mathrm{bf}]-[126 \mathrm{bg}]+[126 \mathrm{~cd}]-[126 \mathrm{cf}]+[13 \mathrm{abf}]-[13 \mathrm{ace}]+[13 \mathrm{acg}]+[13 \mathrm{bcf}] \\
& -[13 \mathrm{bcg}]+[14 \mathrm{ace}]-[15 \mathrm{abf}]+[16 \mathrm{bcd}]-[16 \mathrm{bcf}]+[23 \mathrm{ace}]-[23 \mathrm{acf}]-[25 \mathrm{abe}]+[25 \mathrm{abf}] \\
& +[25 \mathrm{bcd}]-[25 \mathrm{bce}]+[26 \mathrm{abe}]-[26 \mathrm{abf}]-[26 \mathrm{ace}]+[26 \mathrm{acf}]-[26 \mathrm{bcd}]+[26 \mathrm{bce}]+[3 \mathrm{acfg}] \\
& -[3 \mathrm{bcfg}]-[4 \mathrm{abcd}]-[5 \mathrm{abde}]+[5 \mathrm{acde}]-[5 \mathrm{bcde}]-[6 \mathrm{abef}]+[6 \mathrm{acef}]-[6 \mathrm{bcef}]
\end{aligned}
$$

The non-realizability follows from the existence of the following positive Plücker tree:

$$
\Gamma(14 \mathrm{bg} \mid 025 \mathrm{a})-[124 \mathrm{abg}]^{?}-\Gamma(14 \mathrm{ag} \mid 02 \mathrm{cf})-[14 \mathrm{abfg}]^{?}--\Gamma(4 \mathrm{abg} \mid 13 \mathrm{cf})
$$

with final certificate

$$
\begin{aligned}
\text { [014ga2] [014bg5] [13agb4] [4abcgf] } & +[014 \mathrm{ga} 2][014 \mathrm{bg} 5][4 \mathrm{abcg} 1][3 \mathrm{abgf} 4] \\
+[014 \mathrm{bg} 2][014 \mathrm{gaf}][15 \mathrm{abg} 4][4 \mathrm{abcg} 3] & +[014 \mathrm{bg} 5][04 \mathrm{abg} 1][124 \mathrm{afg}][4 \mathrm{abcg} 3] \\
& +[04 \mathrm{abg} 1][014 \mathrm{gaf}][125 \mathrm{bg} 4][4 \mathrm{abcg} 3] .
\end{aligned}
$$

Remark 5.8. In our search, we have only optimized over the number of nodes in the tree, not over the number of facets that are involved. This accounts for the different numbers of relevant facets in Theorems 5.4-5.7. We have not attempted to find the minimal number of relevant facets in each case.

Remark 5.9. For none of the four prismatoids have we found non-realizability certificates that only use the "first" and "last" layer [CS19].

Remark 5.10. We would like to insist on the fact that irrespectively of the machine computations that have gone into finding these certificates, the fact that they are valid is perfectly checkable by humans.

5.3. Jockusch's 3 -spheres. Recall Jockusch's family of 3 -spheres, whose member $\Delta_{6}^{3}$ was shown to be non-realizable in Proposition 1.2.

Theorem 5.11. For $n \geq 5$, no member $\Delta_{n}^{3}$ of Jockusch's family of 3-spheres is polytopal.

Proof. The facets of $\Delta_{n}^{3}$ are listed explicitly in [NZ20, Lemma 3.1], and among them we find a certain combinatorial 3-ball $\pm B_{n}^{3,1}$. Moreover, this 3-ball is the only subcomplex of $\Delta_{n}^{3}$ that gets deleted in the inductive construction of $\Delta_{n+1}^{3}$ from $\Delta_{n}^{3}$. Therefore, to prove our claim for $n \geq 6$ it suffices to check that no determinant in the Plücker certificate from Proposition 1.2 involves the facets of $\pm B_{6}^{3,1}$, which are

[1256] [1 $\overline{1} \overline{2} \overline{5} \overline{6}][\overline{1} \overline{2} 56][12 \overline{5} \overline{6}][2356][\overline{2} \overline{3} \overline{5} \overline{6}] \quad[\overline{2} \overline{3} 56][23 \overline{5} \overline{6}][3456]$

$[\overline{3} \overline{4} \overline{5} \overline{6}][\overline{3} \overline{4} 56][34 \overline{5} \overline{6}][1 \overline{4} 56][\overline{1} 4 \overline{5} \overline{6}][1 \overline{4} \overline{5} 6][\overline{1} 45 \overline{6}][1 \overline{4} \overline{5} \overline{6}][\overline{1} 456]$.

Unfortunately, the certificate of Proposition 1.2 does contain, for example, the forbidden facet $[1 \overline{4} \overline{5} \overline{6}]$ ! We therefore need a different certificate. The provably smallest useful tree certificate is

$$
\begin{array}{r}
{[12 \overline{6} \overline{4} \overline{2}]([\overline{1} \overline{2} \overline{6} \overline{4} 1]([23 \overline{6} \overline{4} 5]([\overline{1} 24 \overline{5} 6]([23 \overline{6} \overline{4} 5]([\overline{1} 2 \overline{3} 43]([12 \overline{6} \overline{4} \overline{1}]([124 \overline{3} \overline{6}] \Gamma(124 \mid 3 \overline{3} \overline{4} 5)} \\
+[124 \overline{3} 3] \Gamma(124 \mid \overline{3} \overline{4} 5 \overline{6})) \\
+[124 \overline{3} 3][124 \overline{3} \overline{4}](-\Gamma(12 \overline{6} \mid \overline{1} 4 \overline{4} 5))) \\
+[124 \overline{3} 3][124 \overline{3} \overline{4}][12 \overline{6} \overline{4} 5] \Gamma(\overline{1} 24 \mid 13 \overline{3} \overline{6})) \\
+[124 \overline{3} 3][124 \overline{3} \overline{4}][12 \overline{6} \overline{4} 4][\overline{1} 2 \overline{3} 43] \Gamma(25 \overline{6} \mid 1 \overline{1} 3 \overline{4})) \\
+[124 \overline{3} 3][124 \overline{3} \overline{4}][12 \overline{6} \overline{4} 5][\overline{1} 2 \overline{3} 4 \overline{6}][23 \overline{6} \overline{4} 5] \Gamma(\overline{1} 24 \mid 13 \overline{5} 6)) \\
+[\overline{1} 2 \overline{3} 41][124 \overline{3} 3][124 \overline{3} \overline{4}][12 \overline{6} \overline{4} 5][\overline{1} 24 \overline{5} 6][23 \overline{6} \overline{4} 5](-\Gamma(23 \overline{6} \mid \overline{1} 4 \overline{4} 5))) \\
+[124 \overline{3} 3][1235 \overline{6}][124 \overline{3} \overline{4}][12 \overline{6} \overline{4} 4][\overline{1} 2 \overline{3} 43][\overline{1} 24 \overline{5} 6][23 \overline{6} \overline{4} 5] \Gamma(\overline{1} \overline{4} \overline{6} \mid 12 \overline{2} 5)) \\
+[124 \overline{3} 3][1235 \overline{6}][124 \overline{3} \overline{4}][12 \overline{6} \overline{4} 4][\overline{1} \overline{2} \overline{6} \overline{4} 2][\overline{1} 2 \overline{3} 43][\overline{1} 24 \overline{5} 6][23 \overline{6} \overline{4} 5](-\Gamma(1 \overline{4} \overline{6} \mid \overline{1} 2 \overline{2} 5)),
\end{array}
$$


which simplifies to

$$
\begin{aligned}
& \quad[\overline{1} 2 \overline{3} 41][\overline{1} \overline{2} \overline{6} \overline{4} 1][12 \overline{6} \overline{4} \overline{2}][124 \overline{3} 3][124 \overline{3} \overline{4}][12 \overline{6} \overline{4} 5][23 \overline{6} \overline{4} \overline{1}][\overline{1} 24 \overline{5} 6][34 \overline{6} 52][23 \overline{6} \overline{4} 5] \\
& +[\overline{1} 2 \overline{3} 41][\overline{1} \overline{2} \overline{6} \overline{4} 1][12 \overline{6} \overline{4} \overline{2}][124 \overline{3} 3][124 \overline{3} \overline{4}][12 \overline{6} \overline{4} 5][\overline{1} 3 \overline{6} 52][\overline{1} 24 \overline{5} 6][23 \overline{6} \overline{4} 4][23 \overline{6} \overline{4} 5] \\
& +[\overline{1} 24 \overline{5} 1][\overline{1} \overline{2} \overline{6} \overline{4} 1][12 \overline{6} \overline{4} \overline{2}][124 \overline{3} 3][124 \overline{3} \overline{4}][12 \overline{6} \overline{4} 5][2364 \overline{1}][\overline{1} 2 \overline{3} 4 \overline{6}][23 \overline{6} \overline{4} 5]^{2} \\
& +[1264 \overline{1}][\overline{1} \overline{2} \overline{6} \overline{4} 1][12 \overline{6} \overline{4} \overline{2}][124 \overline{3} 3][124 \overline{3} \overline{4}][12 \overline{6} \overline{4} 5][234 \overline{5} \overline{1}][\overline{1} 2 \overline{3} 4 \overline{6}][23 \overline{6} \overline{4} 5]^{2} \\
& +[12 \overline{6} \overline{4} \overline{1}][\overline{1} \overline{2} \overline{6} \overline{4} 1][12 \overline{6} \overline{4} \overline{2}][124 \overline{3} 3][124 \overline{3} 5][12 \overline{6} \overline{4} 4][\overline{1} 2 \overline{3} 43][\overline{1} 24 \overline{5} 6][23 \overline{6} \overline{4} 5]^{2} \\
& +[12 \overline{6} \overline{4} \overline{1}][\overline{1} \overline{2} \overline{6} \overline{4} 1][12 \overline{6} \overline{4} \overline{2}][12 \overline{4} 34][124 \overline{3} 5][124 \overline{3} \overline{6}][\overline{1} 2 \overline{3} 43][\overline{1} 24 \overline{5} 6][23 \overline{6} \overline{4} 5]^{2} \\
& +[12 \overline{6} \overline{4} \overline{1}][12 \overline{6} \overline{4} \overline{2}][124 \overline{3} 3][1235 \overline{6}][124 \overline{3} \overline{4}][12 \overline{6} \overline{4} 4][\overline{1} 2 \overline{3} 43][\overline{1} 24 \overline{5} 6][\overline{1} \overline{2} \overline{6} \overline{4} 5][23 \overline{6} \overline{4} 5] \\
& +[12 \overline{6} \overline{4} \overline{1}][124 \overline{3} 3][1235 \overline{6}][124 \overline{3} \overline{4}][12 \overline{6} \overline{4} 4][1 \overline{2} \overline{4} 5 \overline{6}][\overline{1} \overline{2} \overline{6} \overline{4} 2][\overline{1} 2 \overline{3} 43][\overline{1} 24 \overline{5} 6][23 \overline{6} \overline{4} 5] \\
& +[\overline{1} \overline{2} \overline{6} \overline{4} 1][12 \overline{6} \overline{4} \overline{2}][124 \overline{3} 3][124 \overline{3} \overline{4}][12 \overline{6} \overline{4} 4][12 \overline{6} \overline{4} 5][\overline{1} 2 \overline{3} 43][\overline{1} 3 \overline{6} 52][\overline{1} 24 \overline{5} 6][23 \overline{6} \overline{4} 5] \\
& +[\overline{1} \overline{2} \overline{6} \overline{4} 1][124 \overline{3} 3][1235 \overline{6}][124 \overline{3} \overline{4}][12 \overline{6} \overline{4} 4][12 \overline{6} \overline{4} 5][\overline{1} \overline{2} \overline{6} \overline{4} 2][\overline{1} 2 \overline{3} 43][\overline{1} 24 \overline{5} 6][23 \overline{6} \overline{4} 5]
\end{aligned}
$$

None of these terms contains any forbidden facet, and the minimality of this certificate is guaranteed by the fact that the integer linear program of Section 4.4 was solved to optimality. The non-realizability of $\Delta_{5}^{3}$ must be checked separately. We omit its certificate, which needs six Plücker polynomials.

Remark 5.12. Jockusch's non-realizable 3 -sphere $\Delta_{5}^{3}$ with 10 vertices has the same $f$-vector $(10,40,60,30)$ as the boundary complex of the 4-polytope $P=\operatorname{conv}\left(\diamond^{4} \cup\{ \pm \mathbf{1}\}\right)$ obtained from the cross-polytope $\diamond^{4}$, but these 3 -spheres are not combinatorially isomorphic. The facets of the non-realizable $\Delta_{5}^{3}$ are

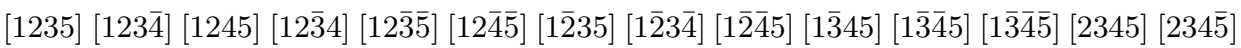

$[23 \overline{4} \overline{5}][\overline{1} 24 \overline{5}][\overline{1} 2 \overline{3} 4][\overline{1} 2 \overline{3} \overline{5}][\overline{1} 345][\overline{1} 34 \overline{5}][\overline{1} 3 \overline{4} \overline{5}][\overline{1} \overline{2} 35][\overline{1} \overline{2} 3 \overline{4}][\overline{1} \overline{2} 45][\overline{1} \overline{2} \overline{3} 4][\overline{1} \overline{2} \overline{3} \overline{5}][\overline{1} \overline{4} \overline{4} \overline{5}][\overline{2} \overline{3} 45]$

$[\overline{2} \overline{3} \overline{4} 5][\overline{2} \overline{3} \overline{4} \overline{5}]$,

while the facets of the realizable 3 -sphere $\partial P$ are

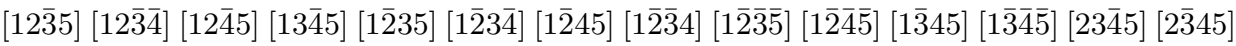

[2 $\overline{3} \overline{4} \overline{5}][\overline{1} 235][\overline{1} 23 \overline{4}][\overline{1} 245][\overline{1} 2 \overline{3} 4][\overline{1} 2 \overline{3} \overline{5}][\overline{1} 2 \overline{4} \overline{5}][\overline{1} 345][\overline{1} 3 \overline{4} \overline{5}][\overline{1} \overline{2} 34][\overline{1} \overline{2} 3 \overline{5}][\overline{1} \overline{2} 4 \overline{5}][\overline{1} \overline{3} 4 \overline{5}][\overline{2} 345]$

$[\overline{2} 3 \overline{4} \overline{5}][\overline{2} \overline{3} 4 \overline{5}]$.

5.4. Novik and Zheng's centrally symmetric neighborly $d$-spheres. In [NZ19], Novik and Zheng give several constructions of centrally symmetric, highly neighborly $d$-spheres. They are based on a family $\Delta_{n}^{d}$ of cs- $\left\lceil\frac{d}{2}\right\rceil$-neighborly combinatorial $d$-spheres on $2 n \geq 2 d+2$ vertices, which arise as the case $i=\left\lceil\frac{d}{2}\right\rceil$ of an inductively constructed family $\Delta_{n}^{d, i}$ of cs- $i$-neighborly combinatorial $d$-spheres. Each of those contains a certain combinatorial $d$-ball $B_{n}^{d, i-1}$, which is the only part that gets deleted in a step of the inductive construction. For $d=3$, Novik and Zheng's family $\left\{\Delta_{n}^{3}: n \geq 4\right\}$ is precisely Jockusch's family from [Joc95], and $B_{n}^{3,1}$ is precisely the ball $B_{n}^{3}$ mentioned in Theorem 5.11.

Theorem 5.13. For $n \geq 6$, no member $\Delta_{n}^{4}$ of Novik and Zheng's family is realizable.

Proof. The construction of $\Delta_{n}^{4}$ in [NZ19, Section 3] can be made explicit as follows [Nov20].

(1) The facets of $B_{n}^{4,1}$ are

(i) $\{i, i+1, n-2, n-1, n\}$ and $\{-i,-i-1, n-2, n-1, n\}$ for $1 \leq i \leq n-4$, and

(ii) $\{1,-n+3, n-2, n-1, n\},\{1,-n+3,-n+2, n-1, n\},\{1,-n+3,-n+2,-n+1, n\}$, $\{1,-n+3,-n+2,-n+1,-n\}$,

(2) The remaining facets of $\Delta_{n}^{4}$ are

(i) $\{i, i+1, \ell-3, \ell-2, \ell\},\{i, i+1, \ell-3, \ell-1, \ell\},\{-i,-i-1, \ell-3, \ell-2, \ell\}$, $\{-i,-i-1, \ell-3, \ell-1, \ell\}$, where $1 \leq i \leq \ell-5 \leq n-5$ (equivalently, $6 \leq i+5 \leq \ell \leq n$ );

(ii) $\{1,-\ell+4, \ell-3, \ell-2, \ell\},\{1,-\ell+4, \ell-3, \ell-1, \ell\},\{1,-\ell+4,-\ell+3, \ell-2, \ell\}$, $\{1,-\ell+4,-\ell+2, \ell-1, \ell\},\{1,-\ell+4,-\ell+2,-\ell+1, \ell\},\{1,-\ell+4,-\ell+3,-\ell+1, \ell\}$, $\{-\ell+4,-\ell+3,-\ell+2, \ell-1, \ell\},\{-\ell+4,-\ell+3,-\ell+2,-\ell+1, \ell\}$, where $6 \leq \ell \leq n ;$

(3) together with the following 10 facets:

$\{-1,2,-3,4,-5\},\{1,2,-3,4,-5\},\{1,2,3,4,-5\},\{1,2,3,-4,-5\},\{1,-2,-3,4,-5\}$,

$\{1,-2,3,4,-5\},\{1,-2,3,-4,-5\},\{-1,-2,-3,4,-5\},\{-1,-2,3,4,-5\},\{-1,-2,3,-4,-5\}$. 
The polymake implementation ${ }^{1}$ of this construction has successfully passed various consistency checks.

As to the non-realizability of these spheres, as in Theorem 5.11 the case $n=6$ has to be dealt with separately, with an omitted certificate consisting of 18 Plücker polynomials. There is, in this case, no certificate that avoids the facets of the balls $\pm B_{6}^{4,1}$.

For $n=7$, we do find a certificate that avoids the facets of $\pm B_{7}^{4,1}$, which are

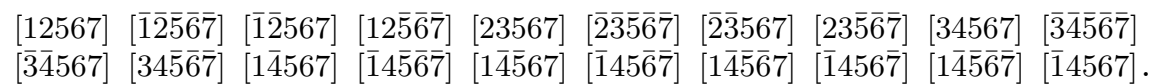

It is provably minimal, and uses the 28 Plücker polynomials

$$
\begin{aligned}
& \Gamma_{0}=-\Gamma(1 \overline{2} \overline{3} 6 \mid 4 \overline{4} 5 \overline{7}), \quad \Gamma_{1}=\Gamma(1 \overline{2} \overline{3} 6 \mid \overline{4} 5 \overline{5} \overline{7}), \quad \Gamma_{2}=\Gamma(1 \overline{2} 56 \mid \overline{3} 4 \overline{4} \overline{7}), \quad \Gamma_{3}=-\Gamma(1 \overline{3} 45 \mid \overline{2} 67 \overline{7}), \\
& \Gamma_{4}=-\Gamma(1 \overline{3} 67 \mid \overline{2} 4 \overline{4} \overline{6}), \quad \Gamma_{5}=\Gamma(\overline{1} \overline{2} \overline{3} 5 \mid \overline{4} \overline{5} 6 \overline{7}), \quad \Gamma_{6}=-\Gamma(\overline{1} \overline{2} \overline{3} \overline{5} \mid 4 \overline{4} 5 \overline{7}), \quad \Gamma_{7}=-\Gamma(\overline{1} \overline{2} \overline{3} 6 \mid 145 \overline{5}), \\
& \Gamma_{8}=\Gamma(\overline{2} 356 \mid 1 \overline{3} 4 \overline{7}), \quad \Gamma_{9}=-\Gamma(\overline{2} 356 \mid \overline{1} \overline{3} 4 \overline{7}), \quad \Gamma_{10}=\Gamma(\overline{2} \overline{3} 45 \mid 1 \overline{1} \overline{5} \overline{7}), \quad \Gamma_{11}=-\Gamma(\overline{2} \overline{3} 46 \mid 1 \overline{1} 35), \\
& \Gamma_{12}=\Gamma(\overline{2} \overline{3} \overline{4} 6 \mid 14 \overline{5} 7), \quad \Gamma_{13}=-\Gamma(\overline{2} \overline{3} \overline{4} 6 \mid 15 \overline{6} \overline{7}), \quad \Gamma_{14}=\Gamma(\overline{2} \overline{3} \overline{4} 6 \mid 1 \overline{5} \overline{6} \overline{7}), \quad \Gamma_{15}=\Gamma(\overline{2} \overline{3} \overline{4} \overline{6} \mid 1 \overline{1} 67), \\
& \Gamma_{16}=-\Gamma(\overline{2} \overline{3} \overline{4} \overline{6} \mid 167 \overline{7}), \quad \Gamma_{17}=\Gamma(\overline{2} \overline{3} 56 \mid 1 \overline{1} 3 \overline{4}), \quad \Gamma_{18}=\Gamma(\overline{2} \overline{3} 56 \mid 13 \overline{4} \overline{5}), \quad \Gamma_{19}=\Gamma(\overline{2} \overline{3} 56 \mid 13 \overline{4} \overline{7}), \\
& \Gamma_{20}=\Gamma(\overline{2} \overline{3} 56 \mid \overline{1} 34 \overline{4}), \quad \Gamma_{21}=\Gamma(\overline{2} \overline{3} 56 \mid \overline{1} 4 \overline{4} \overline{7}), \quad \Gamma_{22}=\Gamma(\overline{2} \overline{3} 56 \mid 3 \overline{4} \overline{5} \overline{7}), \quad \Gamma_{23}=\Gamma(\overline{2} \overline{3} \overline{5} \overline{7} \mid 14 \overline{4} 5), \\
& \Gamma_{24}=-\Gamma(\overline{2} \overline{3} \overline{5} \overline{7} \mid 1 \overline{4} 56), \quad \Gamma_{25}=-\Gamma(\overline{2} \overline{3} 67 \mid 4 \overline{4} \overline{5} \overline{6}), \quad \Gamma_{26}=\Gamma(\overline{3} 6 \overline{6} 7 \mid 1 \overline{2} \overline{4} \overline{5}), \quad \Gamma_{27}=-\Gamma(456 \overline{7} \mid 1 \overline{2} 3 \overline{3})
\end{aligned}
$$

arranged as in Figure 2. Since it contains no facet of $\pm B_{7}^{4,1}$, it survives the inductive construction.

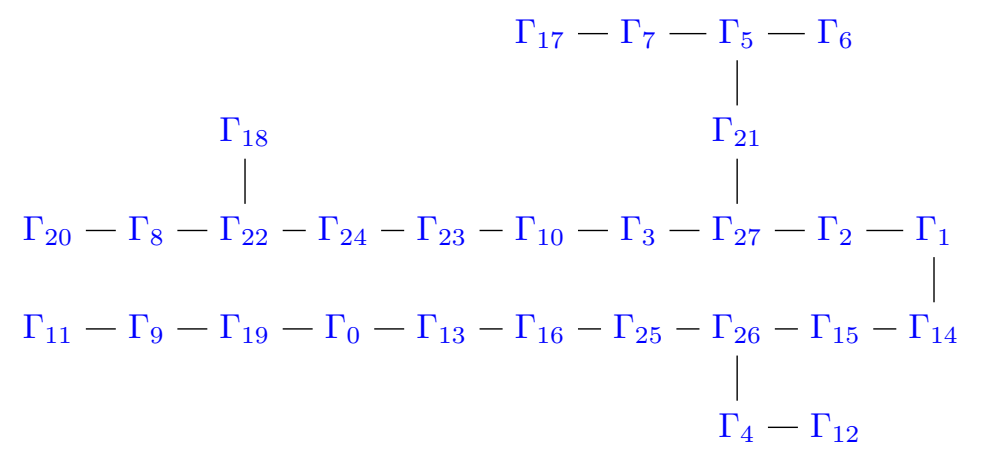

Figure 2. The minimal Plücker tree proving the non-realizability of $\Delta_{7}^{4}$

Theorem 5.14. [Zhe20b] For $n-2 \geq d \geq 3$, no member $\Delta_{n}^{d}$ of Novik and Zheng's family is realizable.

Proof. By [NZ19, Proposition 4.1], each sphere $\Delta_{n}^{d}$ occurs as a face link in $\Delta_{n+2}^{d+2}$ and $\Delta_{n+3}^{d+3}$. Since links of realizable spheres are realizable, Theorem 5.11 yields the proof for all $n \geq d+2$ and $d \geq 5$, and Theorem 5.13 directly settles the remaining case $d=4$.

Remark 5.15. In [NZ20], Novik and Zheng describe several other families of highly neighborly centrally symmetric spheres. Once explicit facet descriptions of these are implemented, they can be checked for realizability using the present methods.

\section{Acknowledgements}

It is a pleasure to thank Francisco Santos, Michael Joswig and Günter M. Ziegler for crucial discussions and their careful reading and pertinent suggestions. Moreover, I am very grateful to Amy Wiebe and Antonio Macchia for finding and pointing out an error in a previous version, and to Isabella Novik and Heilun Zheng for stimulating discussions, and for pointing out various consequences of this work to their families of cs-neighborly spheres.

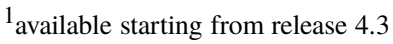




\section{REFERENCES}

[Alo86] Noga Alon. The number of polytopes, configurations and real matroids. Mathematika, 33:62-71, 1986.

[AP17] Karim Adiprasito and Arnau Padrol. The universality theorem for neighborly polytopes. Combinatorica, 37:129-136, 2017.

[BS89] Jürgen Bokowski and Bernd Sturmfels. Computational synthetic geometry, volume 1355. Berlin etc.: Springer-Verlag, 1989.

[BW19] Madeline Brandt and Amy Wiebe. The slack realization space of a matroid. Algebr. Comb., 2(4):663-681, 2019.

[CS19] Francisco Criado and Francisco Santos. Topological Prismatoids and Small Simplicial Spheres of Large Diameter. Experimental Mathematics, 2019. https:doi.org/10.1080/10586458.2019.1641766.

[DId17] Mareike Dressler, Sadik Iliman, and Timo de Wolff. A Positivstellensatz for sums of nonnegative circuit polynomials. SIAM J. Appl. Algebra Geom., 1(1):536-555, 2017.

[DMP12] Jesús A. De Loera, Peter N. Malkin, and Pablo A. Parrilo. Computation with polynomial equations and inequalities arising in combinatorial optimization. In Mixed integer nonlinear programming. Selected papers based on the presentations at the IMA workshop mixed-integer nonlinear optimization: Algorithmic advances and applications, Minneapolis, MN, USA, November 17-21, 2008, pages 447-481. New York, NY: Springer, 2012.

[GJ00] Ewgenij Gawrilow and Michael Joswig. polymake: a framework for analyzing convex polytopes. In Polytopescombinatorics and computation (Oberwolfach, 1997), volume 29 of DMV Sem., pages 43-73. Birkhäuser, Basel, 2000.

[GMTW19] João Gouveia, Antonio Macchia, Rekha R. Thomas, and Amy Wiebe. The Slack realization space of a polytope. SIAM J. Discrete Math., 33(3):1637-1653, 2019.

[GMW20] João Gouveia, Antonio Macchia, and Amy Wiebe. Combining realization space models of polytopes, 2020, 2001.11999.

[GP86] Jacob E. Goodman and Richard Pollack. Upper bounds for configurations and polytopes in $\mathbb{R}^{d}$. Discrete Comput. Geom. 1:219-227, 1986.

[JM18] Martina Juhnke-Kubitzke and Satoshi Murai. Balanced generalized lower bound inequality for simplicial polytopes. Sel. Math., New Ser., 24(2):1677-1689, 2018.

[JMNS18] Martina Juhnke-Kubitzke, Satoshi Murai, Isabella Novik, and Connor Sawaske. A generalized lower bound theorem for balanced manifolds. Math. Z., 289(3-4):921-942, 2018.

[Joc95] William Jockusch. An infinite family of nearly neighborly centrally symmetric 3 -spheres. J. Comb. Theory, Ser. A, 72(2):318-321, 1995.

[Kal88] Gil Kalai. Many triangulated spheres. Discrete Comput. Geom., 3(1-2):1-14, 1988.

[Mne88] Nikolai E. Mnev. The universality theorems on the classification problem of configuration varieties and convex polytopes varieties. Topology and geometry, Rohlin Semin. 1984-1986, Lect. Notes Math. 1346, 527-543 (1988)., 1988.

[MS05] Ezra Miller and Bernd Sturmfels. Combinatorial commutative algebra, volume 227. New York, NY: Springer, 2005.

[MS15] Diane Maclagan and Bernd Sturmfels. Introduction to tropical geometry, volume 161. Providence, RI: American Mathematical Society (AMS), 2015.

[Mun84] James R. Munkres. Elements of Algebraic Topology. Addison-Wesley, 1984.

[Nov20] Isabella Novik. personal communication, 2020.

[NSW16] Eran Nevo, Francisco Santos, and Stedman Wilson. Many triangulated odd-dimensional spheres. Math. Ann., 364(34):737-762, 2016.

[NZ19] Isabella Novik and Hailun Zheng. Highly neighborly centrally symmetric spheres, 2019, 1907.06115.

[NZ20] Isabella Novik and Hailun Zheng. New families of highly neighborly centrally symmetric spheres, 2020, 2005.01155.

[PPS12] Julian Pfeifle, Vincent Pilaud, and Francisco Santos. Polytopality and Cartesian products of graphs. Isr. J. Math., 192:121-141, 2012.

[PZ04] Julian Pfeifle and Günter M. Ziegler. Many triangulated 3-spheres. Math. Ann., 330(4):829-837, 2004.

[RZ95] Jürgen Richter-Gebert and Günter M. Ziegler. Realization spaces of 4-polytopes are universal. Bull. Am. Math. Soc., New Ser., 32(4):403-412, 1995.

[San12] Francisco Santos. A counterexample to the Hirsch conjecture. Ann. Math. (2), 176(1):383-412, 2012.

[Sho91] Peter W. Shor. Stretchability of pseudolines is NP-hard. In A dual forest algorithm for the assignment problem, pages 531-554. 1991

[Sta75] Richard P. Stanley. The upper bound conjecture and Cohen-Macaulay rings. Stud. Appl. Math., 54:135-142, 1975.

[Stu02] Bernd Sturmfels. Solving systems of polynomial equations, volume 97. Providence, RI: American Mathematical Society (AMS), 2002.

[Ven19] Lorenzo Venturello. Balanced triangulations on few vertices and an implementation of cross-flips. Electron. J. Comb., 26(3):research paper p3.61, 25, 2019.

[Wie20] Amy Wiebe. personal communication, 2020.

[Zhe20a] Hailun Zheng. Ear decomposition and balanced neighborly simplicial manifolds. Electron. J. Comb., 27(1):research paper p1.10, 17, 2020.

[Zhe20b] Hailun Zheng. personal communication, 2020.

Departament de Matemàtica Aplicada, Universitat Politècnica de Catalunya

Email address: julian.pfeifle@upc.edu 